\title{
Live Imaging
}

\author{
George Chaconas ${ }^{1 *}$, Tara J. Moriarty ${ }^{2}$, Jon Skare ${ }^{3}$ and Jenny A. Hyde ${ }^{3}$ \\ ${ }^{1}$ Department of Biochemistry and Molecular Biology and Department of Microbiology, Immunology and Infectious \\ Diseases, Snyder Institute for Chronic Diseases, University of Calgary, Calgary, Alberta, T2N 4N1, Canada \\ ${ }^{2}$ Faculties of Dentistry and Medicine (Laboratory Medicine and Pathobiology), University of Toronto, Toronto, \\ Ontario, M5G 1G6, Canada \\ ${ }^{3}$ Department of Microbial Pathogenesis and Immunology, Texas A \& M University Health Science Center, Bryan, \\ Texas, 77807, USA \\ *Corresponding author: chaconas@ucalgary.ca
}

DOI: https://doi.org/10.21775/cimb.042.385

\begin{abstract}
Being able to vizualize a pathogen at a site of interaction with a host is an aesthetically appealing idea and the resulting images can be both informative as well as enjoyable to view. Moreover, the approaches used to derive these images can be powerful in terms of offering data unobtainable by other methods. In this article, we review three primary modalities for live imaging Borrelia spirochetes: whole animal imaging, intravital microscopy and live cell imaging. Each method has strengths and weaknesses, which we review, as well as specific purposes for which they are optimally utilized. Live imaging borriliae is a relatively recent development and there was a need of a review to cover the area. Here, in addition to the methods themselves, we also review areas of spirochete biology that have been significantly impacted by live imaging and present a collection of images associated with the forward motion in the field driven by imaging studies.
\end{abstract}

\section{Introduction}

Imaging has become one of the primary methods for the analysis of pathogen-host interactions and imaging of living cells and organisms has recently made a large contribution to our knowledge of $B$. burgdorferi pathogenesis. The focus on imaging of live pathogens and or host cells/animals can provide powerful in situ results on dynamic interactions that are not obtainable using spirochetes grown in culture or analyzed in vitro or after tissue fixation. This article reviews currently available technologies for live imaging that have been used on $B$. burgdorferi and that have revealed significant new findings (Radolf and Samuels, 2021). We start with the view from 10,000 feet, which allows visualization of $B$. burgdorferi infection of the entire animal. This is followed by a lower altitude sighting of specific organ systems or anatomical sites by high resolution, realtime imaging of live spirochetes in a living animal. Finally, we focus on live imaging in cellular systems. Each approach can provide unique and important information. The major contributions that these methodologies have made to our current understanding of Lyme disease pathogenesis will be reviewed. This article cannot provide a comprehensive review of all the topics covered here, and non-imaging data from the papers cited below is not discussed; instead, the reader is referred to the original literature for complete details of the papers we examine.

The methodologies to be described here are highly varied, as are the processes studied and the resulting knowledge gained. Whole animal imaging makes use of bioluminescent probes and has been successfully used to study Borrelia pathogenesis including dissemination and colonization of the mouse using a variety of $B$. burgdorferi mutant strains. By coupling specific gene promoters to luminescent probes, the methodology has also proven useful to study the localized expression in the mouse of several spirochete genes following infection. Intravital microscopy has opened a new area of study in terms of $B$. burgdorferi-endothelial interactions, the innate immune response to $B$. burgdorferi infection, vascular transmigration, the localized effectiveness of antibiotic treatment in the mouse and $B$. burgdorferi motility and chemotaxis 
during infection. Intravital imaging allows the study of these processes at specific locations in a living mouse at high resolution and in real-time. Finally, live cell imaging provides the highest resolution analysis and has allowed for novel, in-depth of study of $B$. burgdorferi-tick and $B$. burgdorferi-mammalian cell interactions by the imaging of dissected whole mount infected tick midguts and salivary glands and $B$. burgdorferi-cell interactions in chambers mimicking vascular flow. Although widely diverse in technical methodology, the approaches described below are tethered to each other through their common use of light to image the desired processes.

\section{Whole Animal Imaging}

Bioluminescent in vivo imaging $(\mathrm{BLI})$ is a widely utilized technique to investigate a variety of questions from tumor development, immune response and host-pathogen interactions to bacterial pathogenesis (Contag et al., 1995; Rehemtulla et al., 2000; Sadikot et al., 2002; Jenkins et al., 2003; Hutchens and Luker, 2007; Lienenklaus et al., 2009; Andreu et al., 2011; $\mathrm{Xu}$ et al., 2016). The first publication of bioluminescent bacteria was Contag et al. (Contag et al., 1995) in Salmonella typhimurium utilizing the luciferase gene from the firefly (Photinus pyralis) to spatiotemporally track infection in mice. This method provides a satellite view of infection as it progresses in mice and is not designed for an assessment of pathogenesis on a cellular level. Bioluminescence is the result of an enzymatic reaction by luciferase with the D-luciferin substrate, oxygen, and ATP (Figure 1). The robust signal is emitted from viable cells with relatively low background. Over the years, a variety of luciferase alleles from insects and marine organisms have been developed to capture light emission from deep tissues within mammalian hosts (Zhang et al., 1999; Waidmann et al., 2011; Xu et al., 2016). To capture images and quantify bioluminescence emission from mammalian tissue, a couple-charged device (CCD) camera is required. $B L I$ was first implemented with $B$. burgdorferi using a constitutively expressed codon optimized Photinus pyralis luciferase gene on a multicopy shuttle vector (designated as Bbluc; (Blevins et al., 2007; Hyde et al., 2011). This work was followed by the development of single copy Bb/uc, a borrelial codon optimized Renilla luciferase (Rluc $\mathrm{C}_{B b}$ ), and several fluorescent proteins with potential use in the murine model (Chan et al., 2015; Adams et al., 2017; Takacs et al., 2018).

\section{Methodology}

Most bioluminescent borrelial strains employ a luciferase gene that is encoded on a shuttle vector and maintained through in vivo selective pressure (Hyde et al., 2011; Wager et al., 2015; Zhi et al., 2015; Skare et al., 2016; Adams et al., 2017). A multicopy plasmid-encoded form of luciferase is necessary for a high level of sensitivity in order to decipher subtle phenotypic differences. To facilitate this, constitutively expressed Bb/uc, expressed from the strong $f l a B$ promoter $\left(\mathrm{P}_{f l a B}\right)$, was introduced into pBBE22 within a strain background that used positive selection for pncA (bbe22) to maintain the presence of the luc gene during infection for up to 91 days post-infection without an added antibiotic (Figure 1; (Hyde et al., 2011; Wager et al., 2015; Zhi et al., 2015; Skare et al., 2016; Medina-Perez et al., 2020; Saputra et al., 2020). It has since been demonstrated that antibiotic supplementation in drinking water can maintain luc-based bioluminescence throughout a 10 day period of murine infection (Adams et al., 2016).

Experimentation using luciferase-based imaging with $B$. burgdorferi has been restricted to needle inoculation of mice although one study evaluated the acquisition of bioluminescent $B$. burgdorferi in crushed tick samples (Adams et al., 2017). Initially, Balb/c mice were used given the low skin content of melanin to maximize the acquired bioluminescent signal (Hyde et al., 2011; Wager et al., 2015; Skare et al., 2016; Saputra et al., 2020). More recently, however, $\mathrm{C} 57$ and $\mathrm{C} 3 \mathrm{H}$ mice have been utilized, albeit at a lower level of sensitivity (Zhi et al., 2015; Medina-Perez et al., 2020). The advantage of needle inoculation is the ability to infect with a known amount of $B$. burgdorferi. To image, the mice are anesthetized using isoflurane and injected intraperitoneally with D-luciferin, the substrate for BbLuc, and then placed in an imager with a CCD camera that can detect light emission (Hyde and Skare, 2018). Following injection of the D-luciferin substrate, the mice can be imaged for up to 45 minutes; however, the signal does proportionally decrease over time. It is important to include a background control mouse in each image for every group of mice and time point for accurate analysis of the quantified bioluminescent signal. There are multiple acceptable approaches to control for background. One approach is to infect all the mice in the group with bioluminescent $B$. burgdorferi and not inject one mouse with D-luciferin (Hyde et al., 2011; Wager et al., 2015; Zhi et al., 2015; Hyde and Skare, 2018; Medina-Perez et al., 2020). Alternatively, one 
A. D-Luciferin
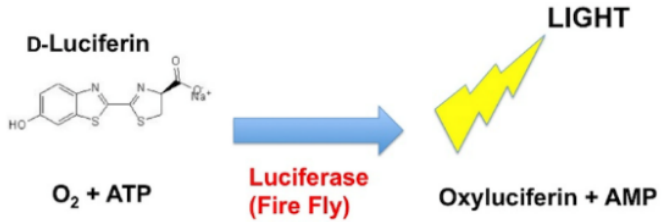

B.

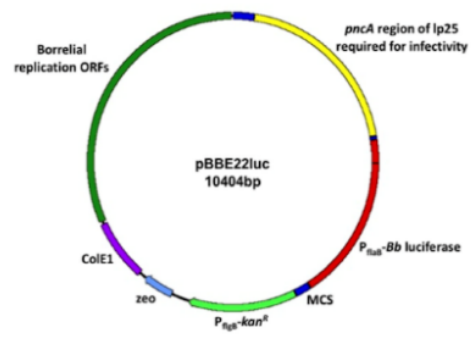

C.

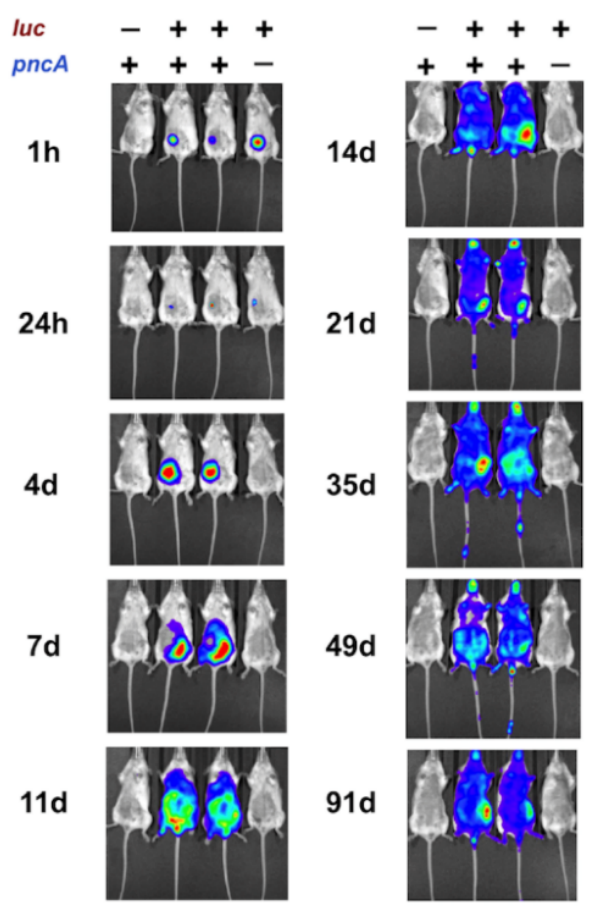

Figure 1. The implementation of bioluminescent imaging (BLI) in Borrelia burgdorferi. (A) Luciferase enzymology. Enzymology of luciferase that results in light formation. This reaction requires ATP and dissolved oxygen. (B) Construction of a luciferase containing plasmid for BLI following infection with B. burgdorferi. The Bbluc gene was cloned into the pBBE22 shuttle vector, which contains the $25 \mathrm{~kb}$ linear plasmid (Ip25) pncA (bbe22) gene, to generate pBBE22/uc. B. burgdorferi strains lacking the Ip25 plasmid are non-infectious. The presence of pncA together with Bbluc promotes simultaneous positive selection for the maintenance of this plasmid during infection and the constitutive expression of Bbluc. (C) In vivo tracking of luciferase-expressing $B$. burgdorferi. A ventral view of Balb/C mice infected with $10^{5} \mathrm{~B}$. burgdorferi constitutively expressing Bbluc. The images are from the same 4 mice imaged at $1 \mathrm{~h}$ out to 91 days (d). All mice imaged were given D-luciferin except the mouse on the left, which is infected with infectious $B$. burgdorferi with no Bbluc gene; the mouse on the right is infected with $B$. burgdorferi containing Bbluc but lacking the $p n c A$ (bbe22) gene required for persistence. The presence of the $p n c A$ and luc genes is indicated at the top left. For the images shown, the signal range averaged between $5.5 \times 10^{3}$ to $1.0 \times 10^{5}$ photons per second $(\mathrm{p} / \mathrm{s})$. 
mouse in each group can be infected with $B$. burgdorferi lacking luciferase (Figure 1) or encoding a promoterless luciferase and then treated with $D$ luciferin as with the other mice in the group.

A challenge in experimental $B$. burgdorferi infection is determining tissue specific localization during later stages of disease. For deeper tissue colonization, mice can be treated with D-luciferin, sacrificed, organs harvested, incubated in additional substrate, and imaged (Skare et al., 2016; Saputra et al., 2020). Imaging of individual tissues allows the direct quantification of bioluminescence as a representation of bacterial burden or gene expression without further manipulation as would be necessary using real-time PCR.

\section{Advantages}

The greatest advantage of whole animal imaging is the ability to track infection of $B$. burgdorferi in a living animal over an extended period of time and observe quantifiable changes in infectivity, dissemination, and gene expression by non-invasive means. Of particular note, the in vivo bioluminescent evaluation of borrelial gene regulation involves environmental conditions that cannot be recapitulated under in vitro conditions or in dialysis membrane chambers incubated in rat peritoneal cavities. The low level of background luminescence, which often plagues fluorescence techniques, contributes to increased sensitivity and detection. Another distinguishing feature from fluorescence is that light emission is an indicator of bacterial viability (Figure 1). The requirement for oxygen and ATP as reaction cofactors for light production is found only in viable cells, whereas a decline in fluorescence indicates degradation of the protein and, therefore, diminished excitability. This sensitivity has proven to be particularly important in the comparison of mutants in various genes relative to the parental and complemented strains (Hyde et al., 2011; Wager et al., 2015; Zhi et al., 2015; Skare et al., 2016; MedinaPerez et al., 2020). Inherent to this, the number of animals required to get an assessment of infection is significantly reduced, and the same animals are repeatedly monitored, providing a less variable and more cohesive kinetic evaluation of infection.

\section{Disadvantages}

$B$. burgdorferi bioluminescent imaging is limited by the strength of the emitted signal, which is dependent on the number of bacteria present, strength of the promoter driving Bb/uc, and the copy number of
Bb/uc. BLI in B. burgdorferi has been utilized primarily with the robust, constitutively expressed flaB promoter driving Bbluc expression encoded on a multicopy shuttle vector (Figure 1). Weaker promoters linked to Bbluc reduce the sensitivity of detection or possibly are not detectable above background levels. The quantifiable range of bioluminescence is 600 to 60,000 counts for valid analyses of bioluminescence imaging (Hyde and Skare, 2018). One other potential issue is the use of a multicopy Bb/uc to visualize light emitting $B$. burgdorferi. To date, bioluminescence driven by flaB or other promoters on a multicopy plasmid has correlated well with borrelial burden or transcript levels, respectively, but this may not be the case for every gene of interest (Hyde et al., 2011; Skare et al., 2016; Saputra et al., 2020).

One issue with whole animal imaging is the lack of resolution of individual bacterial cells; that is, the signal only indicates localization within the skin in a broad sense as organisms disseminate intracutaneously (Waidmann et al., 2011). In vivo imaging of the whole animal or individual tissues provides limited insight into the molecular mechanisms of pathogenesis. Other techniques outlined in this review involve individual $B$. burgdorferi cells interacting in vivo with the host vasculature and tissues. Another limitation of whole animal imaging is the inability to detect and quantify $B$. burgdorferi in deeper tissues; thus, signals in tissues such as the heart, bladder, tibiotarsal joint, and lymph nodes are not accurate. As indicated above, and outlined in more detail below, organs can be imaged ex vivo to determine borrelial localization, but this requires invasive techniques and more mice if several time points are needed (Skare et al., 2016; Saputra et al., 2020).

\section{Phenotypic characterization of luciferase expressing B. burgdorferi strains}

The ability to detect light-emitting $B$. burgdorferi has provided important insight into how Lyme disease spirochetes can establish and maintain an infection in a small cohort of mice both temporally and spatially (Hyde et al., 2011). This technology was used initially to assess the phenotype of a $B$. burgdorferi bbk32 mutant that exhibited an apparent conflicting phenotype in the literature (Li et al., 2006; Seshu et al., 2006). The differences observed between these three week end point studies was attributed to the infectious dose used; specifically, when a high and non-physiologically relevant load was used (e.g., $10^{5}$ 


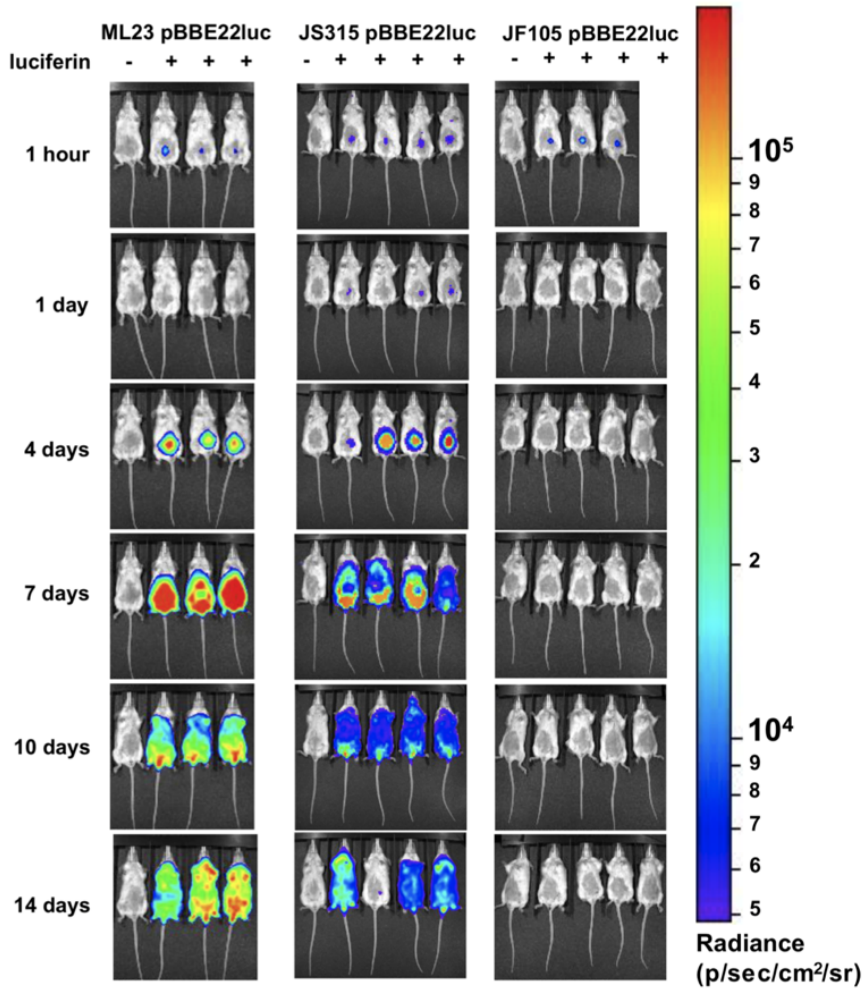

Figure 2. Tracking phenotypes of $B$. burgdorferi mutants using BLI. Infectivity of the wild type parent strain (ML23 pBBE22/uc), a B. burgdorferi bbk32 mutant (JS315 pBBE22/uc), and a B. burgdorferi dbpBA deletion (JF105 pBBE22/uc) were compared in Balb/c mice. Mice were infected intradermally at $10^{3}$ $B$. burgdorferi and imaged. The mouse on the far left is infected equivalently as the others but was not given D-luciferin substrate (denoted with a "minus"). Mice given substrate are indicated with a "plus". All images for each time point and strain shown were standardized to the same photon per second range. This figure is reproduced from (Hyde et al., 2011; Zhi et al., 2015) with permission from John Wiley and Sons, Inc.

spirochetes), very little difference was observed between the infection of the $B$. burgdorferi bbk32 mutant relative to its parent (Li et al., 2006; Seshu et al., 2006). However, when a lower and more physiological infectious dose was used (e.g., $10^{3}$ spirochetes) a significant difference in infectivity was observed (Seshu et al., 2006). When the $B$. burgdorferi bbk32 mutant was transformed with the Bb/uc construct (Figure 2), the resulting data demonstrated an attenuated phenotype at both the lower and higher dose and provided additional quantitative infection kinetics of the $B$. burgdorferi bbk32 mutant (Hyde et al., 2011). Specifically, the overall signal was significantly less starting at 7 days and was 4.1- to 7.7-fold less at all time points (Hyde et al., 2011). For the $10^{5}$ dose, the light signal for the
B.burgdorferi bbk32 mutant was 3-fold less than that for the parent strain at day 7 (Hyde et al., 2011).

Imaging values also correlated well with qPCR. The $B$. burgdorferi bbk32 mutant showed significantly reduced bacterial burdens by qPCR in the skin, lymph node, and joint, independent of dose (Hyde et al., 2011). Total genomic B. burgdorferi DNA from infected skin correlated well with bioluminescent imaging, indicating that the detection of light emitting $B$. burgdorferi is a valid approach for quantifying borrelial burdens during active infection (Hyde et al., 2011). This method also has the added advantage of deciphering subtle differences in infectivity not otherwise apparent without tracking genomic equivalents via qPCR at discrete time points. While a 
valuable and valid means to determine infectivity, qPCR at multiple time points has the disadvantage of being expensive, laborious, and, most importantly, requires a large number of animals to obtain relevant data.

The experiments discussed above used multicopy Bb/uc to detect light emitting $B$. burgdorferi following infection. Another group integrated the Bb/uc gene and a linked antibiotic resistance locus into the strain N40 bbe02 restriction/modification system gene (Chan et al., 2015). By virtue of its construction, this strain was more competent for transformation and gained bioluminescence. When imaged, however, the signal detected was not uniform between the mice tested, presumably due to the low inoculum doses used $\left(10,100\right.$, and $\left.10^{3}\right)$, which were, in some instances, at or below the $50 \%$ infectious dose for $B$. burgdorferi (ID50).

Having established bioluminescent imaging as a method to characterize and quantify $B$. burgdorferi infectivity, numerous subsequent studies used this approach to track mutant phenotypes in live mice. In a manner similar to the $B$. burgdorferi bbk32 mutant, the inactivation of $b b 0744$ (p83/100), as well as the non-coding RNA itt $A$, resulted in a significantly attenuated phenotype relative to the infectious parent strain (Wager et al., 2015; Medina-Perez et al., 2020). Other mutants that are severely impaired in their ability to cause experimental infection, including $B$. burgdorferi $d b p B A$ and bba33, were vetted using bioluminescence, either confirming (for $d b p B A$ mutants) or demonstrating (for bba33) their loss of virulence (Blevins et al., 2008; Shi et al., 2008; Weening et al., 2008; Hyde et al., 2011; Zhi et al., 2015). Typical images of "wildtype" (parent), subtle, or pronounced phenotypic differences are shown in Figure 2. Note that other borrelial genes have been targeted but did not yield an infectivity defect via imaging or other metrics of infection (unpublished). These analyses reinforced the strength of this approach, notably, that a phenotype, be it mild or profound, can be evaluated in a limited but significant pool of experimentally infected mice in a sensitive and timely manner.

Use of imaging to track the expression of B. burgdorferi genes by transcriptional reporters

$B$. burgdorferi undergoes complex gene regulation in the mammalian host and BLI enables spatiotemporal tracking of changes in gene expression. Promoters for ospC and $d b p B A$ were linked to luciferase, $\mathrm{P}_{\text {osp }} C^{-}$ luc and $\mathrm{P}_{d b p B A}-l u c$, respectively, to monitor the borrelial response to the distinct stages of pathogenesis (Figures 3 and 4) (Skare et al., 2016; Saputra et al., 2020). Bioluminescent reporter strains were normalized for changes in borrelial burden quantified from the constitutively expressed luciferase strain $\mathrm{P}_{\text {flas }}-\mathrm{luc}$. These studies clearly

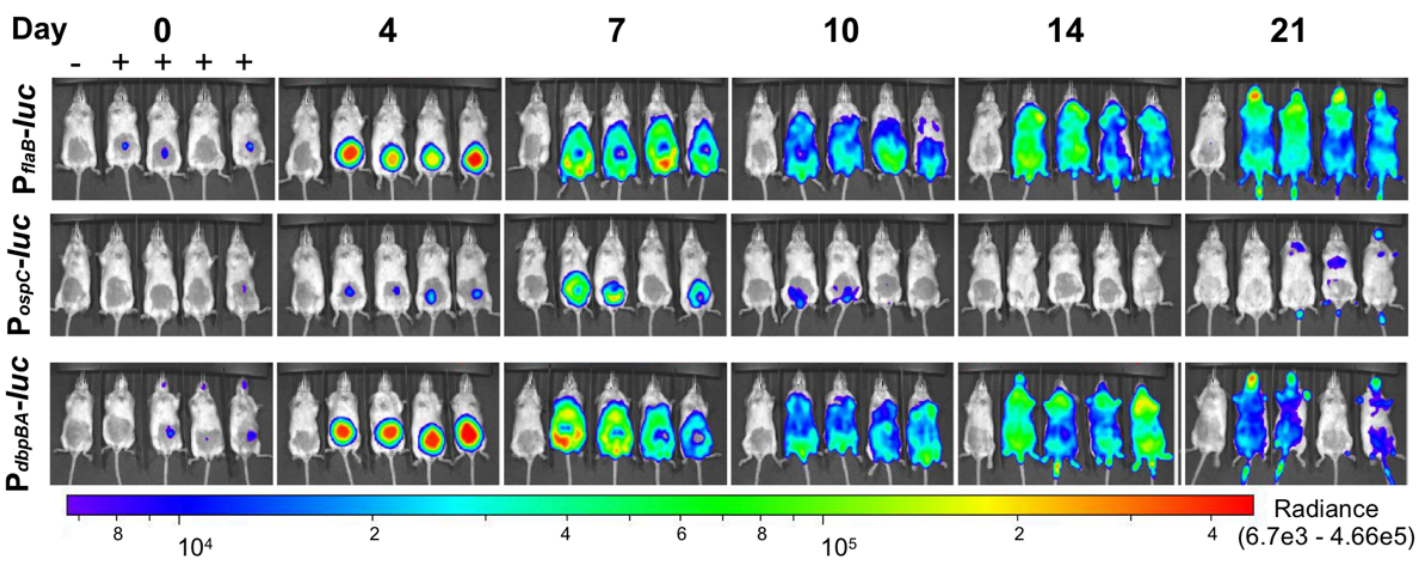

Figure 3. Tracking B. burgdorferi gene expression in infected mice using distinct reporter constructs. Imaging of infected mice with various reporters. Balb/c mice were infected with $10^{5}$ infectious B. burgdorferi containing $\mathrm{P}_{\text {flaB }}-l u c, \mathrm{P}_{\text {ospc-luc, or }} \mathrm{P}_{\mathrm{dbp} B A}-\mathrm{luc}$ reporters and imaged at the time points indicated. The control mouse ("-") was treated as described in Figure 2. All reporters were normalized for interstrain comparison to the spectral emission scale shown below the images. Images reproduced from (Skare et al., 2016; Saputra et al., 2020). 

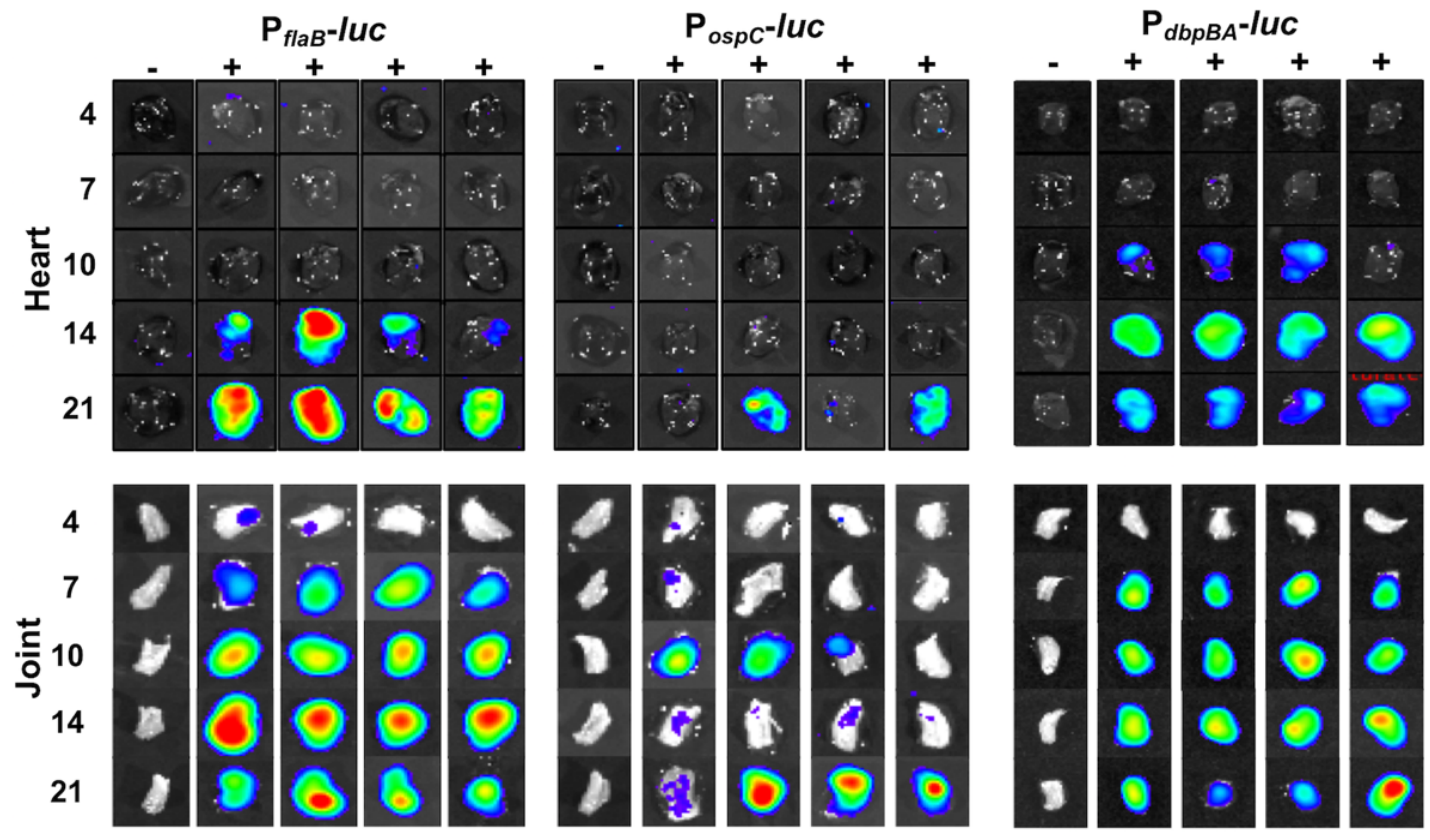

Figure 4. Ex vivo gene expression in tissues in B. burgdorferi infected mice. (A) Imaging of reporter constructs in infectious $B$. burgdorferi within heart and joint tissues. Heart and tibiotarsal joint from mice infected with infectious $B$. burgdorferi containing $P_{f l a B}-l u c, P_{o s p c}-l u c$, or $P_{d b p B A}-l u c$ were imaged

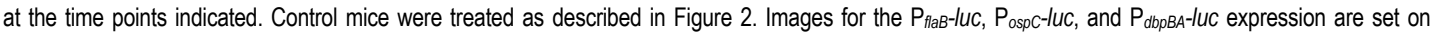
individual scales to display the full spectrum of bioluminescence. Images reproduced from (Skare et al., 2016; Saputra et al., 2020).

demarcated the unique expression patterns of ospC and $d b p B A$ despite both belonging to the RpoS regulon (Hubner et al., 2001; Caimano et al., 2004; Yang et al., 2005; Ouyang et al., 2012; Skare et al., 2016; Saputra et al., 2020). $d b p B A$ was robustly expressed throughout infection, at times exceeding emission from $\mathrm{P}_{\text {flaB }}$-luc (Figure 3) (Saputra et al., 2020). Given the long held paradigm that ospC is needed early in infection (Grimm et al., 2004; Tilly et al., 2006; Tilly et al., 2009), the observation that ospC expression was detectable 21 days post-infection, was surprising (Skare et al., 2016). Imaging of individual tissues demonstrated distinct tissue specific expression that changed over the course of infection. These results demonstrated that ospC and $d b p B A$ are induced in the skin and heart early in infection after which expression declines at these sites (Figure 4). Consistent with the aforementioned atypical late expression of $o s p C$, ospC levels were higher later in the infectious process in the bladder and joint, suggesting that OspC may be important in secondary colonization at these sites or persistence within these tissues. In the inguinal lymph node and tibiotarsal joint, $d b p B A$ reached the highest level of expression after 21 days. Interestingly, ospC was not detectable in the lymph node. These studies highlight the utility of in vivo imaging to quantify the differential expression patterns of targeted $B$. burgdorferi genes in a tissue tropic fashion.

In addition to tracking the expression of known borrelial genes, luciferase based reporters have been used to characterize promoters that are induced following experimental infection of mice. Several of the promoters tested in this way were identified using the BbIVET "promoter trap" approach designed to identify promoters that function during infection (Ellis et al., 2013). This system uses randomly cloned fragments of $B$. burgdorferi DNA to drive expression of pncA (bbe22), an essential gene for borrelial infectivity. Several of the promoters isolated, including those that drive primary, internal, or antisense transcripts were re-tested using the Bb/uc allele (Adams et al., 2017). These studies confirmed 
that in vivo reporters can be utilized in $B$. burgdorferi to study genes spatially and temporally expressed in vivo and, further, track quantifiable differences in live, experimentally infected mice.

\section{Intravital Microscopy Methodology}

Intravital microscopy (IVM) allows the direct visualization of a pathogen at work in a living host, a goal sought for many years and that provides a gold standard for pathogen-host interactions. IVM has been used for decades to investigate a variety of biological processes in living animals (Secklehner et al., 2017). However, its use to investigate bacterial pathogen-host interactions is a relatively recent development with the first report appearing in 2005 (Laschke et al., 2005). An important feature of this methodology is being able to discriminate between the pathogen and the infected host. This is typically accomplished by fluorescent labelling of the pathogen, either by staining or, preferably, by expression of a fluorescent marker, such as GFP, coupled with staining of host cells with fluorescent antibodies or other fluorescent markers. Finally, accessibility of the tissue desired for imaging is an important feature of this methodology, which usually requires surgical preparation of the area of interest prior to imaging.

Early intravital imaging was performed using standard epifluorescence microscopy. More recently, however, more sophisticated microscopes providing superior resolution and imaging are available. Current microscopes of choice are either the spinning disk laser confocal microscope (SDLCM) (Oreopoulos et al., 2014) or a confocal multi-photon microscope (MPM) (Larson, 2011). The spinning disk allows imaging through multiple pinholes, thereby blocking out of focus light from both the excitation and emission light paths. The SDLCM allows high resolution imaging and rapid image acquisition. In contrast, MPM uses near infrared light where the sum of two or more photons reaching the sample provides the energy required to excite the fluorophore. The long wavelength light reduces light scattering and allows deep imaging into the sample and reduces photodamage. MPM also allows direct imaging of certain highly ordered molecular structures (eg collagen) without a fluorophore, through second harmonic generation. In this process, two near infrared photons merge their energies into a single emitted photon of visible light. Confocal microscopes of both SDLCM and MPM types also can be used for $3 \mathrm{D}$ reconstructions, allowing a more realistic analysis of processes in living organisms. Microscopes for intravital imaging need to be equipped with an appropriate stage, objectives and equipment for stabilizing an anesthetized living mouse for microscopic observation (Moriarty et al., 2008; Masedunskas et al., 2012; Belperron et al., 2018).

\section{Advantages}

The major advantage of IVM is that it allows for the direct visualization of a live pathogen in a living organism at high resolution and in real-time. Digital video images can be acquired and quantification is possible in terms of fluorescence intensity, velocity measurements and enumeration of spirochetes. Mechanistic studies are possible by coupling the imaging with genetics, injection of peptides, antibodies and small or macromolecules. Multichannel fluorescence experiments and 3D reconstructions are routinely performed. Combining intravital imaging with the above modalities offers an unprecedented glimpse of pathogenic mechanisms in a variety of locations.

\section{Disadvantages}

IVM is labor intensive and usually requires a high level of surgical skill with mice or other small animals and appropriate state-of-the-art microscopes specifically setup for intravital imaging. It relies on sites that can be effectively imaged (eg, skin, lung, liver, heart, spleen, brain, cremaster) (Secklehner et al., 2017) and the surgical expertise required to prepare each tissue for imaging. Intravital imaging typically requires a large number of pathogens to be present in order to locate and image the infecting organism. In the case of $B$. burgdorferi, IVM may require infection modalities that differ from tick inoculation and may therefore be subject to perturbations from a natural infection. Clarity and resolution can suffer sometimes from small movements due to respiration by the mouse. Finally, the methodology is definitely not high throughput.

\section{B. burgdorferi-endothelial interactions}

Hematogenous dissemination is an important, but poorly understood, facet of Lyme disease pathogenesis (Wormser, 2006). By disseminating to a variety of organ systems, $B$. burgdorferi can cause a wide variety of clinical manifestations and symptoms. But how do spirochetes manage to grab hold of the vascular endothelium in the presence of the extremely strong shear forces found in the 


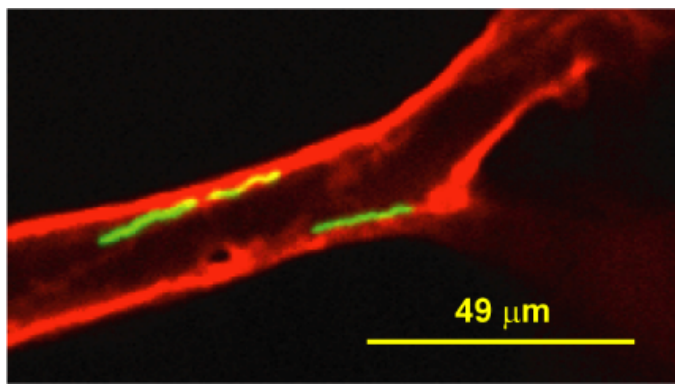

Figure 5. Intravital microscopy to study B. burgdorferi-microvascular interactions. Spinning disk confocal microscopy of GFP-expressing, infectious $B$. burgdorferi in a post-capillary venule of a living mouse. The vasculature was visualized with an AlexaFluor555-conjugated antibody to CD31 (PECAM-1). Image from (Norman et al., 2008).

bloodstream and then cross the endothelium to infect the parenchyma? This process is not effectively or realistically studied outside of the mouse, and it was the starting point for intravital imaging of $B$. burgdorferi by Moriarty and coworkers (Moriarty et al., 2008). The experimental system used continues to provide valuable information on $B$. burgdorferiendothelial interactions and on the vascular transmigration process.

In the first Borrelia intravital imaging experiments, a high concentration of $B$. burgdorferi expressing GFP from a shuttle vector was injected directly into the bloodstream via the jugular or femoral veins of anesthetized mice (resulting in about $10^{7}$ spirochetes $/ \mathrm{ml}$ in the blood). Spirochetes were also visible in the ear at two weeks post-infection; however, this paper focused on $B$. burgdorferivascular interactions immediately after inoculation, when significant numbers of organisms could be observed. The vasculature was labelled with fluorescent CD31 (PECAM-1) antibody; a representative micrograph is shown in Figure 5 . This work showed that, similar to leukocytes (Filippi, 2019), spirochetes engage in multi-stage interactions with the endothelium, including transient tethering interactions, short-lived dragging interactions and stationary adhesion to the vasculature (Figure 6). The vast majority of short-term interactions occurred on the surface of endothelial cells, while $75 \%$ of stationary adhesions were localized to endothelial junctions, suggesting that transmigration might be a paracellular process. Escaping spirochetes were present only at about $0.1 \%$ of the observed spirochete-endothelial interactions and were, therefore, difficult to reliably observe with any confidence. 3D reconstructions also were performed to accurately analyze placement of spirochetes within or outside the observed post-capillary venules. An average displacement velocity of $3.4 \mu \mathrm{m} / \mathrm{min}$ (range 1.1-8.1) was measured for $B$. burgdorferi escaping from post-capillary venules.

A second IVM study of $B$. burgdorferi-vascular interactions by the same group (Norman et al., 2008) focused on mechanistic issues involved in the pathogen-host endothelial interactions and coupled a variety of experimental approaches with IVM. These included the use of gain of function genetic
INITIATION (tethering and draging interactions) --essential first step of microvascular interactions --dependent upon BBK32, GAGs and Fn --may involve other host and B. burgdorferi proteins
STATIONARY ADHESION, EXTRAVASATION

--preferential localization to endothelial junctions --intimate association with PECAM-1 layer of endothelium (stationary adhesions) --rapid, end-first triphasic escape process involving spirochete motility --requires P66 but not BBK32

EXTRAVASCULAR

Figure 6. Schematic summarizing multi-stage interactions of $B$. burgdorferi with the mouse microvasculature based upon IVM (Moriarty et al., 2008; Norman et al., 2008). Tethering and dragging interactions comprise the essential first step of interactions with the microvasculature and are mechanistically distinct from each other regarding BBK32 interactions with Fn and GAGs, respectively (Moriarty et al., 2012). The tethering and dragging interactions are also mechanistically divergent from subsequent stationary adhesions. Stationary adhesion requires additional host and or spirochete factors and result in a redistribution of most spirochetes from cellular locations to endothelial junctions. Vascular transmigration requires P66 (Kumar et al., 2015). Figure modified from (Norman et al., 2008). 


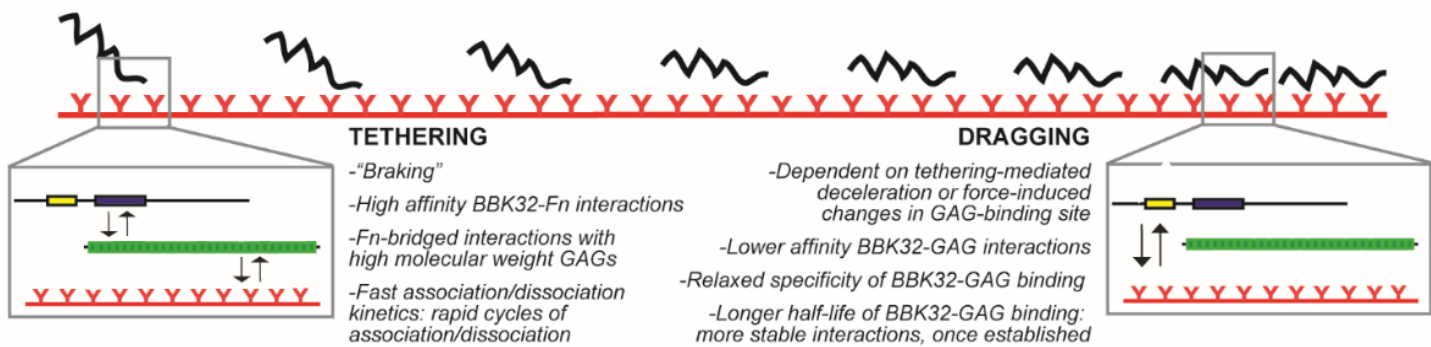

口BBK32 GAG binding sequences BBK32 Fn binding sequences $\mathrm{Y}$ glycosylated endothelial receptors plasma $\mathrm{Fn}$

Figure 7. BBK-mediated tethering and dragging steps of vascular adhesion through distinct macromolecular interactions involving distinct Fn and GAG binding regions. Figure from (Moriarty et al., 2012), with permission from John Wiley and Sons, Inc.

constructs as well as antibodies, peptides and macromolecules to block $B$. burgdorferi-endothelial interactions. Tethering and dragging interactions were shown to be mechanistically distinct from stationary adhesions. Initial interactions were found to be mediated by host fibronectin (Fn) and glycoaminoglycans (GAGs), macromolecules known to interact with a variety of bacterial pathogens, and by the $B$. burgdorferi $\mathrm{Fn}$ and GAG binding protein BBK32. A subsequent IVM study further dissected the role of BBK32 by using BBK32 specifically reduced in either Fn or GAG binding (Moriarty et al., 2012). These studies showed that $\mathrm{Fn}$ binding by BBK32 is responsible for tethering interactions which provide the first point of contact for spirochetes careening through the vasculature under shear force. This molecular braking permits subsequent BBK32GAG interactions that mediate spirochete dragging (Figure 7). This study also showed that RevA, RevB and BB0347 did not provide adhesion activity under shear stress in gain of function strains and that another unknown adhesin (Adhesin X) imparts strong adhesive properties to $B$. burgdorferi in the absence of BBK32. Further characterization of BBK32endothelial interactions is discussed in the live cell imaging section.

The $B$. burgdorferi gain of function vascular adhesion system described above has also been used to assess the endothelial adhesion of Tp0751 (Pallilysin) from Treponema pallidum by expression of this protein in B. burgdorferi followed by IVM (Kao et al., 2017). Pallilysin was found to strongly interact with post-capillary venules in living mice and was also characterized by live cell imaging as noted in that section below.

The innate immune response to $\mathrm{B}$. burgdorferi

IVM has also been used to study the hepatic intravascular immune response to $B$. burgdorferi (Lee et al., 2010). Imaging of the liver after intravenous injection of $B$. burgdorferi revealed that Kupffer cells ingested the spirochetes and induced iNKT cell clustering via CXCR3. Subsequently, direct contact between Kupffer cells and iNKT cells through the antigen-presenting molecule CD1D resulted in iNKT cell activation that reduced $B$. burgdorferi dissemination specifically into the knee joint and surrounding tissue.

A follow-up study by the same group (Lee et al., 2014) used multichannel spinning-disk intravital microscopy of joints and found iNKT cells adjacent to and surrounding the peripheral joint vasculature (Figure 8). The iNKT cells were positioned to ambush spirochetes at the vessel wall and interrupted transmigration of the spirochetes into the joints. $B$. burgdorferi that escaped iNKT cell immune surveillance and transmigrated into the joint tissue were subjected to attack by extravascular iNKT cells (Figure 9), which killed the spirochetes using a granzyme-dependent pathway. The combined results suggest that iNKT cells comprise a potent immune surveillance system and cytotoxic barrier for $B$. burgdorferi transmigration into joint tissue. This is further supported by the large increase in $B$. burgdorferi found specifically in joint tissue in Cd1d-/mice, which are deficient in iNKT cells. This finding 


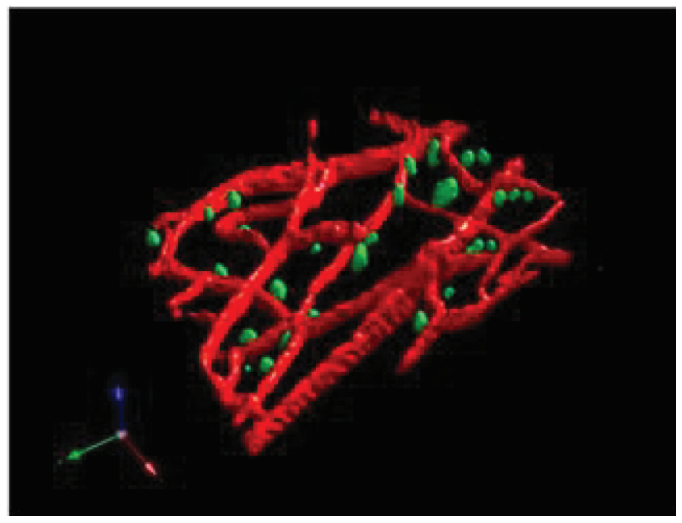

Figure 8. 3D reconstruction of iNKT cells in the mouse joint from IVM images. The iNKT cells are green (CXCR6-GFP, where most GFPexpressing extravascular cells were iNKT cells) and the vasculature red (stained with AlexaFluor555-conjugated antibody to PECAM-1. Image reprinted from (Lee et al., 2014).

was important for the development of an intravital assay to monitor the transmigration ability of $B$. burgdorferi (Kumar et al., 2015) as described in a subsequent section.
Antibiotic treatment of B. burgdorferi-infected mice Two-photon confocal IVM has been used to visualize GFP-expressing $B$. burgdorferi in the dermis and calcaneal tendon of needle-inoculated and tickinfected Myd88-/- mice (Bockenstedt et al., 2012). The use of Myd88-/- mice deficient in the Toll-like receptor adaptor molecule MyD88 results in up to a 100 -fold increase in spirochete burden (Barthold et al., 1992; Bolz et al., 2004; Liu et al., 2004; Wang et al., 2004) and, therefore, facilitates microscopic observation. Using real-time IVM, the authors reported that most $B$. burgdorferi were found moving along collagen fibers in the ear as can be seen in Figure 10. Treatment with ceftriaxone resulted in a dramatic disappearance of spirochetes in the dermis and tendons 24 hours after starting antibiotic treatment (Figure 11). Interestingly, at this time, two morphological transformations of elongated spirochetes into spherical forms were captured by video imaging. A very important finding from this study was that, although antibiotic treatment with ceftriaxone or doxycycline effectively killed $B$. burgdorferi, antigenic spirochete remnants were observed between 2 and 10 weeks after completion of treatment. Spirochete immunogenic and inflammatory antigens also were found in the joints of antibiotic-treated mice, suggesting that they might
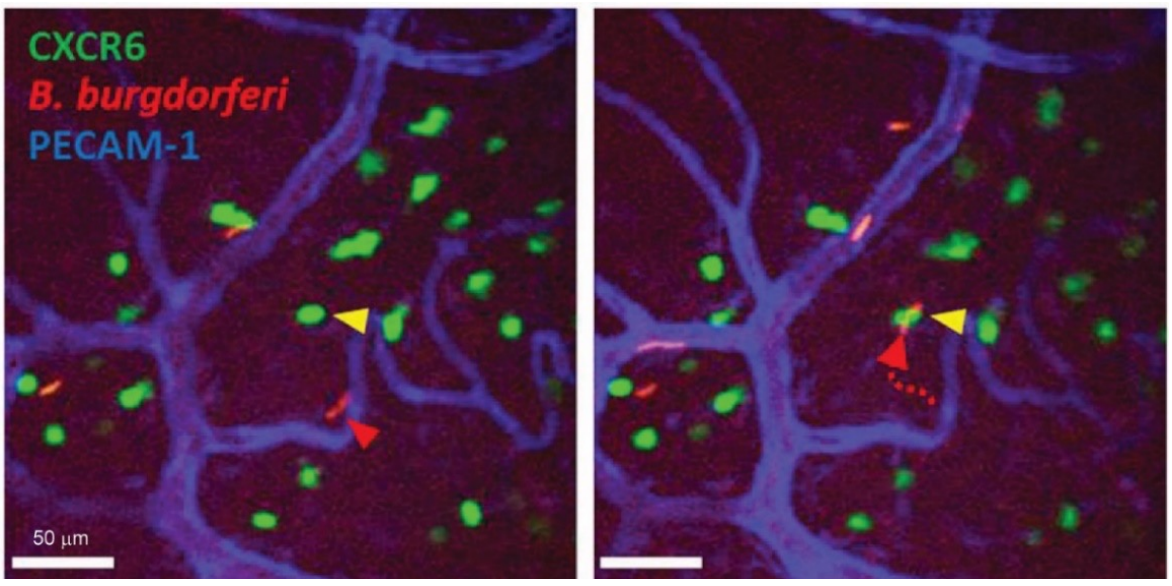

Figure 9. Spirochete attack by an extravascular iNKT cell in CXCR6-GFP mouse joint where most GFP-expressing extravascular cells were iNKT cells. The image on the left shows a motile tomato-labelled extravascular spirochete (red arrow) and a stationary iNKT cell (yellow arrow). The right image shows the path of spirochete migration (red dots) and the spirochete captured by the iNKT cell. The captured spirochete was rendered immobile for the remainder of the experiment (1 hour). Image reprinted from (Lee et al., 2014). 


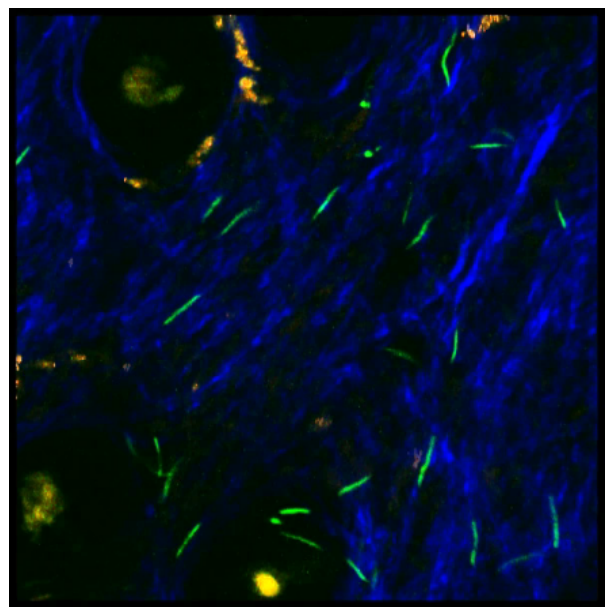

Figure 10. IVM of $B$. burgdorferi expressing GFP in the ear of an immunodeficient mouse 8 weeks post-infection, visualized by confocal two photon microscopy. Second harmonic generation of collagen results in a blue signal, melanin autofluoresces in all channels and appears white/orange, and the hair follicles auto fluoresce yellow (see Belperron et al., 2018). Image from Alexia Belperron and Linda Bockenstedt. play a role in triggering the development of Lyme arthritis and/or other autoimmune sequelae.

\section{Motility and chemotaxis of B. burgdorferi}

The ability of Lyme disease spirochetes to disseminate in a vertebrate host is dependent upon their impressive flagella-driven motility (Sultan et al., 2013; Motaleb et al., 2015; Sultan et al., 2015). This motility has been studied by two-photon confocal IVM in the dermis of mouse ears following tick inoculation (Harman et al., 2012). Spirochete motility was observed to be similar to that previously reported in liquid and in gelatin. However, previously unobserved stationary but motile states were found. These were referred to as wriggling and lunging (Figure 12). The former remained at a fixed location but displayed movement as an undulating, traveling wave. The latter spirochetes were fixed at only one end while they underwent bending and relaxation in apparent attempts to free themselves from their attachment point. These stationary spirochetes are presumed to be tethered to the extracellular matrix by interactions strong enough to withstand the forces of spirochete motility. The IVM observations were compared with $B$. burgdorferi motility in gelatin and media to help establish in vitro conditions for studying spirochete motility that closely matched motility in the mouse dermis.
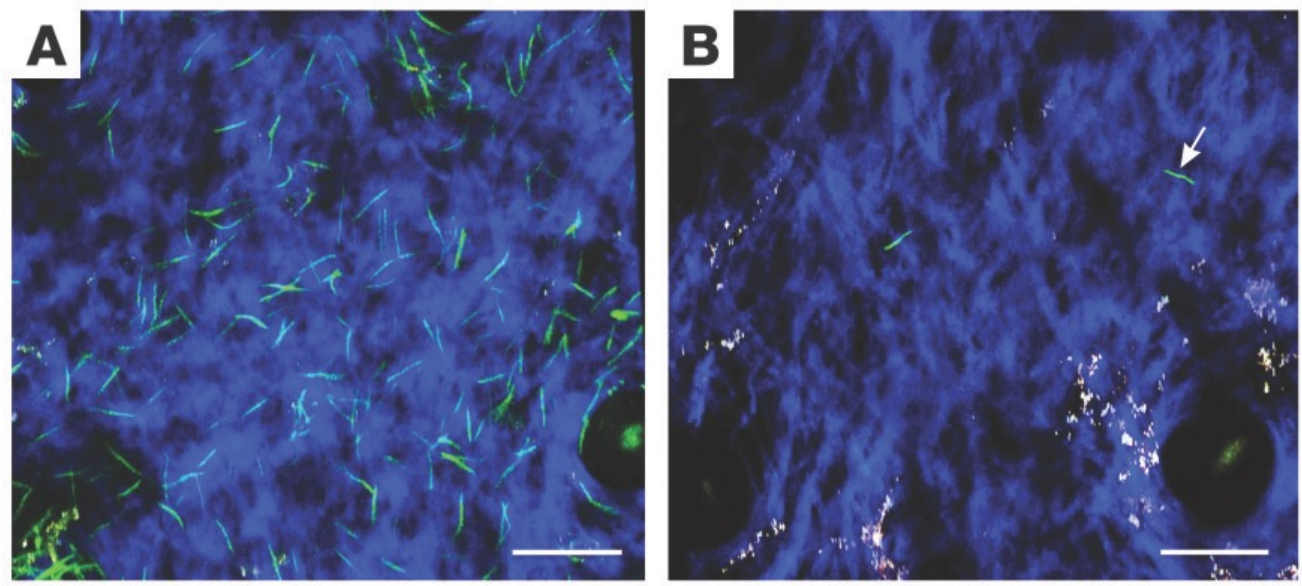

Figure 11. A) B. burgdorferi expressing GFP in skin of a MyD88-/- mouse 22 days post-infection. The blue signal is from second harmonics of skin collagen. B) A dramatic decrease in spirochete numbers is shown after one day of ceftriaxone treatment. The arrow denotes a spirochete that subsequently became a spherical form (not shown). Images from (Bockenstedt et al., 2012), reprinted with permission from the American Society for Clinical Investigation. 

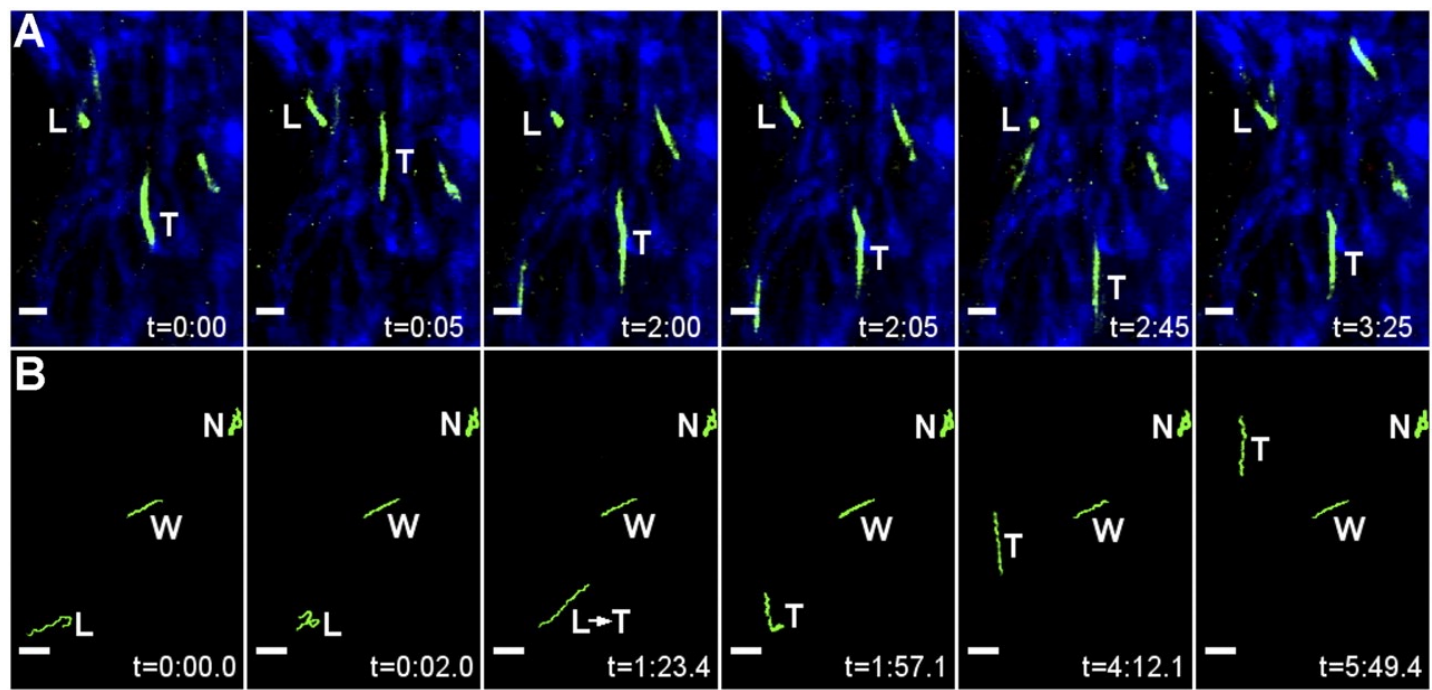

Figure 12. A time course of GFP-expressing spirochete motility patterns in mouse dermis and gelatin. A) Tick-inoculated mouse dermis. B) $3 \%$ gelatin. Lunging $(\mathrm{L})$, translocating $(\mathrm{T})$, wriggling $(\mathrm{W})$ and non-motile $(\mathrm{N})$ spirochetes. The blue signal is from second harmonics of skin collagen. The scale bar is $10 \mu \mathrm{m}$. This figure is reprinted from (Harman et al., 2012).

Two photon IVM microscopy also has been used to visualize Ixodes scapularis feeding on mice in real time and to study the response of nearby $B$. burgdorferi to the feeding ticks (Bockenstedt et al., 2014). Either rag-/- mice, lacking an acquired immune response, or MyD88-/- mice were infected either by needle inoculation or by infestation with infected nymphs. For IVM, unfed nymphs were placed on the ears of mice infected for at least two weeks with GFP-expressing $B$. burgdorferi. The characteristic motility behavior of the observed spirochetes was the same in wild-type, rag-/- and MyD88-/- mice. Restructuring of collagen fibers proximal to the bite site was observed and, although some spirochetes seemed unaffected by the presence of a feeding tick, others were observed to migrate in the direction of the hypostome, suggesting a chemotactic response. This work also supports the currently held belief that spirochetes are acquired from the skin rather than from the blood during tick feeding.

IVM has also been used to study the role of the chemotaxis regulator CheY3 (Novak et al., 2016). B. burgdorferi (wild-type or spirochetes with a disrupted cheY3 gene) were injected intradermally into the dorsal surface of the mouse ear. In comparison to wild-type $B$. burgdorferi, $\Delta$ cheY3 spirochetes displayed little translational motility in the mouse skin at six hours post-infection, with a progressive loss in motility over 48 hours. The spirochetes that were motile at early times displayed translational motility velocities similar to wild-type spirochetes but were unable to reverse direction and continued to run in the same direction (Figure 13). The inability of spirochetes tethered to the ECM to reverse direction and free themselves may be responsible for their loss of motility. $\triangle c h e Y 3$ spirochetes apparently were trapped near the inoculation site and, by 96 hours post-infection, all mutant spirochetes were cleared, while wild-type $B$. burgdorferi increased in numbers. The cheY spirochetes did not disseminate to other tissues. The IVM results clearly demonstrated an essential role for CheY3 in motility, viability and dissemination in the mouse.

\section{Vascular transmigration by $\mathrm{B}$. burgdorferi}

Little is currently known about the vascular transmigration step of $B$. burgdorferi dissemination. Data suggesting a paracellular path whereby spirochetes cross the endothelium at endothelial cell junctions have been reported (Comstock and Thomas, 1989, 1991). In contrast, data suggesting that transmigration occurs via a transcellular pathway 

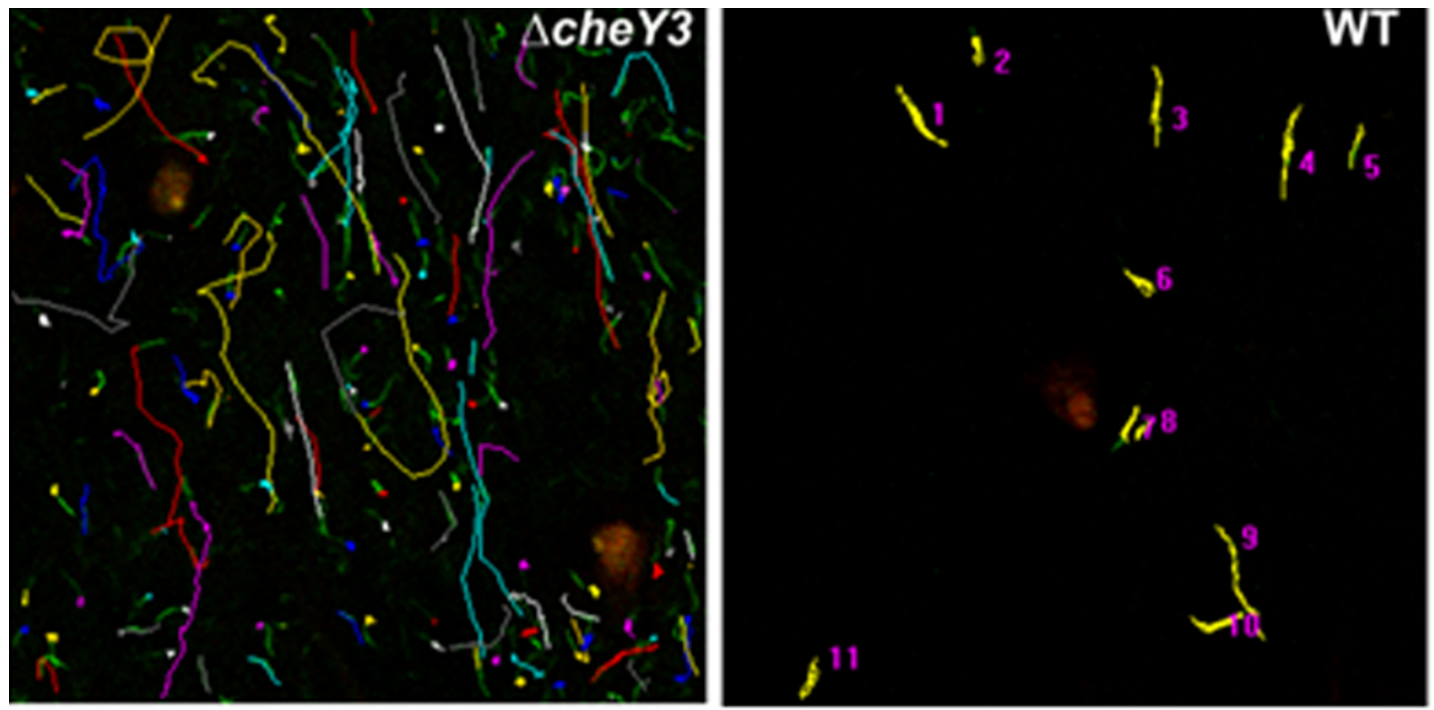

Figure 13. Contrasting motility behavior of $d c h e Y 3$ and WT B. burgdorferi in mouse ear. Paths were tracked at six hours post-injection. Colored lines (except green) show the migration paths of the spirochete. Images from (Novak et al., 2016), with permission from John Wiley and Sons, Inc.
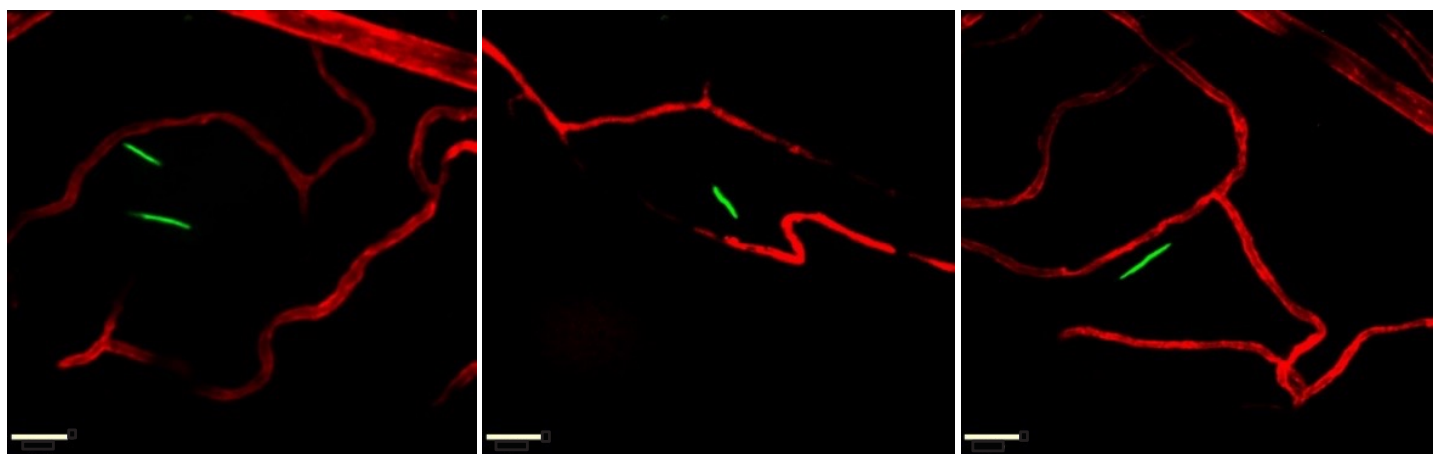

Figure 14. Intravital microscopy to study B. burgdorferi vascular transmigration. Spinning disk confocal microscopy of GFP-expressing, infectious $B$. burgdorferi that escaped from the vasculature in the knee joint-proximal tissue of a living mouse. The vasculature was visualized with an AlexaFluor555conjugated antibody to CD31 (PECAM-1). Image from (Kumar et al., 2015).

have also been reported (Szczepanski et al., 1990). Moreover, little is known regarding the bacterial and host proteins and processes involved. The transmigration process was difficult to study by IVM using using the approach described above for vascular adhesion because looking for transmigrating spirochetes $(0.1 \%$ of observed spirochetes) is akin to looking for a needle in a haystack. Moreover, transmigrated spirochetes rapidly leave the site of vascular escape in the skin, leaving no record. In addition, host defense systems can interfere with transmigration at several levels and $B$. burgdorferi genes involved in survival can be mistaken for genes playing a direct role in transmigration. 


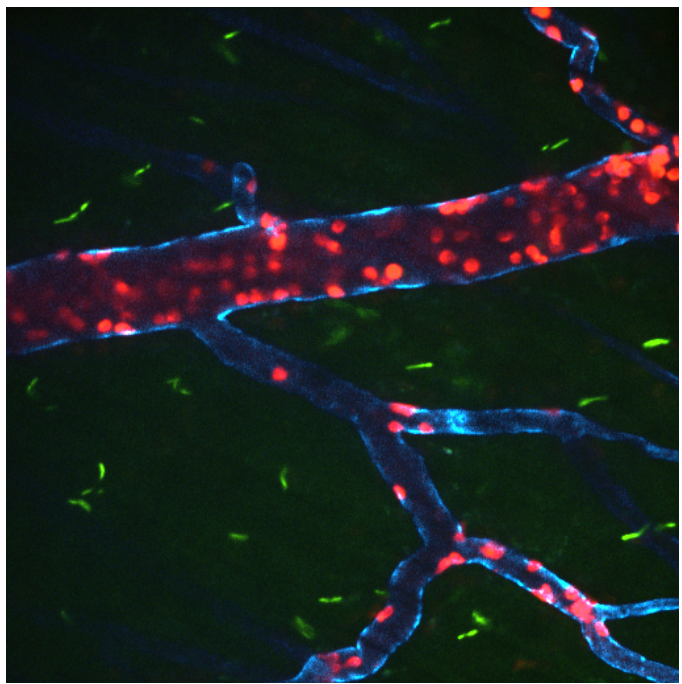

Figure 15. Spinning disk laser confocal micrograph showing endothelial activation in the murine cremaster microvasculature 24 hours after iv injection of GFP-expressing B. burgdorferi. Adherent and crawling neutrophils, physically distanced from spirochetes, were stained were with phycoerythin-conjugated anti-Ly6G (red) and venules were stained with Alexa Fluor 647-conjugated anti-PECAM1 (blue). See (Tan et al., 2020). Image from Björn Petri, Xi Tan and George Chaconas.

To circumvent these problems, an IVM transmigration assay was developed using information discovered in the study of the host innate immune response to $B$. burgdorferi described above (Lee et al., 2010; Lee et al., 2014). The assay uses the peripheral knee vasculature as the site of observation in $\mathrm{Cd} 1 \mathrm{~d}-/$ mice, which lack iNKT cells. Use of Cd1d-/- mice is a crucial feature in this assay system as iNKT cells are the first line of defense against $B$. burgdorferi in mouse knee joint tissue (Lee et al., 2010; Lee et al., 2014). In wild-type mice, spirochetes are rapidly eliminated and unavailable for observation and enumeration. Moreover, the knee joint provides a stable environment where spirochetes remain in the local milieu and can be accurately counted after transmigration. Using this system, spirochetes were injected in the tail vein and visualized in knee joint tissue at 24 hours post-infection (Kumar et al., 2015); very little transmigration occurs before that time. Transmigrated spirochetes are clearly discernible from the vasculature (Figure 14) and display a reciprocating, translational motion outside the vessels. This IVM system was coupled with mutations in the genes encoding the $\mathrm{Fn}$ and GAG binding protein BBK32 and the integrin adhesin and porin, P66. Wild-type levels of transmigration were observed when bbk32 was disrupted; however, disruption of $p 66$ resulted in a loss of transmigration. In particular, mutation of residues involved in integrin binding but not affecting the porin function abrogated transmigration, indicating a role for P66 and integrin binding in vascular transmigration of $B$. burgdorferi. In contrast, disruption of P66 did not influence vascular adhesion, suggesting that initial establishment of a borrelial-endothelial interaction is dependent upon BBK32 and perhaps other $B$. burgdorferi adhesins.

An important component of these IVM experiments was the monitoring of spirochete clearance from the vasculature. The removal of surface adhesins can affect the rate of spirochete clearance. This can result in an apparent decrease in transmigration that stems from a decrease in the number of spirochetes in the vasculature rather than an actual transmigration defect (Kumar et al., 2015).

More recently (Lin et al., 2020), another Borrelia adhesin has been shown to be required for vascular transmigration using IVM. The extracellular matrix binding activity of OspC is specific for fibronectin and dermatan sulphate, and ECM binding by OspC is required for extravasation. OspC does not appear to confer strong vascular adhesion, as BBK32 does, but likely interacts with the vascular endothelium, as P66 does, to promote transmigration subsequent to early tethering and dragging interactions that deaccelerate the spirochetes under the shear stress of bloodflow. The mechanism(s) by which P66 and OspC promote vascular transmigration is unknown at this time, however, it is tempting to speculate that they may induce endothelial signaling to promote cellular changes required for spirochetal transmigration.

Finally, a very recent IVM study (Tan et al., 2020) has revealed that the 24 hour lag observed for vascular transmigration of $B$. burgdorferi results from a requirement for endothelial activation to facilitate vascular escape. Extravasation is stimulated by a factor of three by host neutrophils, which do not recognize or clear the infecting spirochetes. The role of neutrophils appears to be the production of TNF- $\alpha$, MCP-1 (CCL2) and IL-10, all of which can activate the endothelium for transmigration of $B$. burgdorferi. Figure 15 shows adherent and crawling neutrophils that do not recognize $B$. burgdorferi in the murine cremaster microvasculature. 

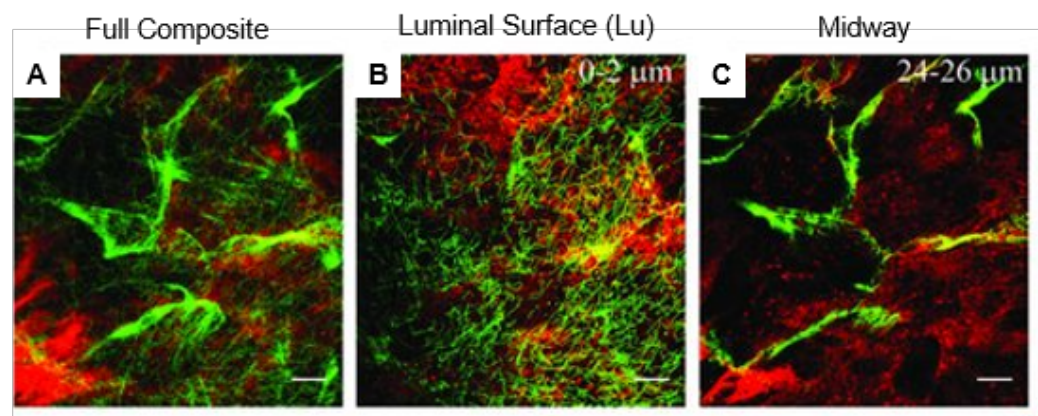

Basement Membrane $(\mathrm{BM})_{\mathrm{yz}}$
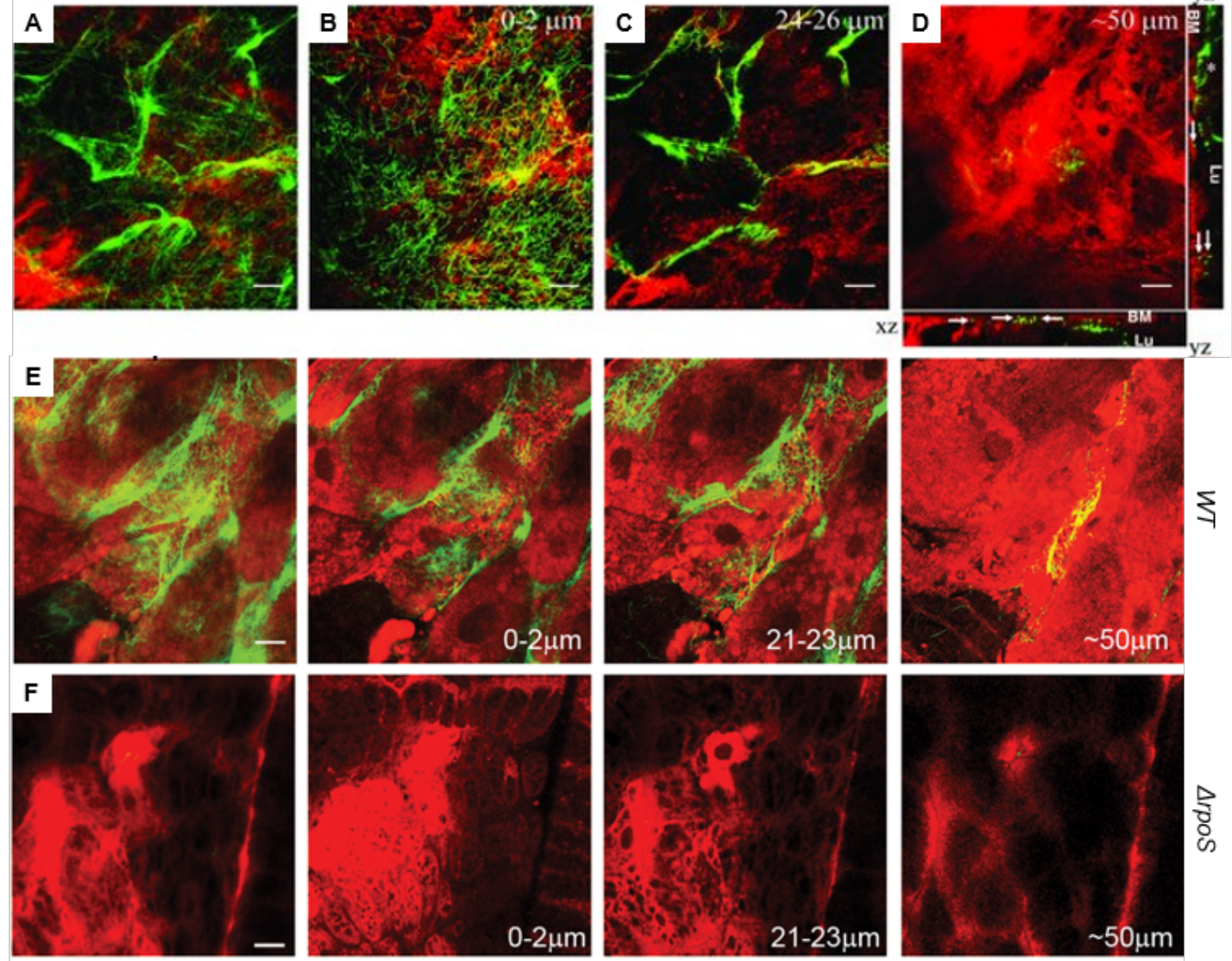

Figure 16. Live ex vivo 3D confocal microscopy z-series of dense, non-motile aggregates of GFP-expressing B. burgdorferi (green) in a hypertrophied region of the midgut epithelium of an infected feeding nymphal tick stained with FM4-64 membrane dye (red). Panel A depicts the composite (projection) image for all optical sections. Panels B-D show single slices obtained at different depths through the thickness of the midgut epithelial lining. $x z$ and $y z$ views of the composite stack are shown at far right/bottom. Panels $\mathrm{E}$ and $\mathrm{F}$ show similar regions from ticks infected with wild-type (WT) and $\Delta r p o S$ GFP-expressing $B$. burgdorferi. Images in A-D from (Dunham-Ems et al., 2009), reprinted with permission from the American Society for Clinical Investigation. Images in E-F from (Dunham-Ems et al., 2012).

\section{Live Cell Imaging Methodologies}

Ex vivo live cell imaging of $B$. burgdorferi-tick interactions Direct observation of $B$. burgdorferi behaviour and biology in ticks is challenging because their cuticle is strongly autofluorescent (Hammer et al., 2001). Multiple approaches have been used to overcome this problem over the past 50 years, including whole organ dissection and cultivation (Grabowski et al., 2018). Current ex vivo imaging methods for studying $B$. burgdorferi-tick interactions visualize GFP- expressing B. burgdorferi in fluorescent counterstained dissected whole mount tick midguts and salivary glands at different stages of the tick blood meal and transmission, using epifluorescence or confocal time lapse microscopy, and confocal optical sectioning for three-dimensional z-series imaging (Figure 16) (Dunham-Ems et al., 2009; Dunham-Ems et al., 2012; Caimano et al., 2015). 

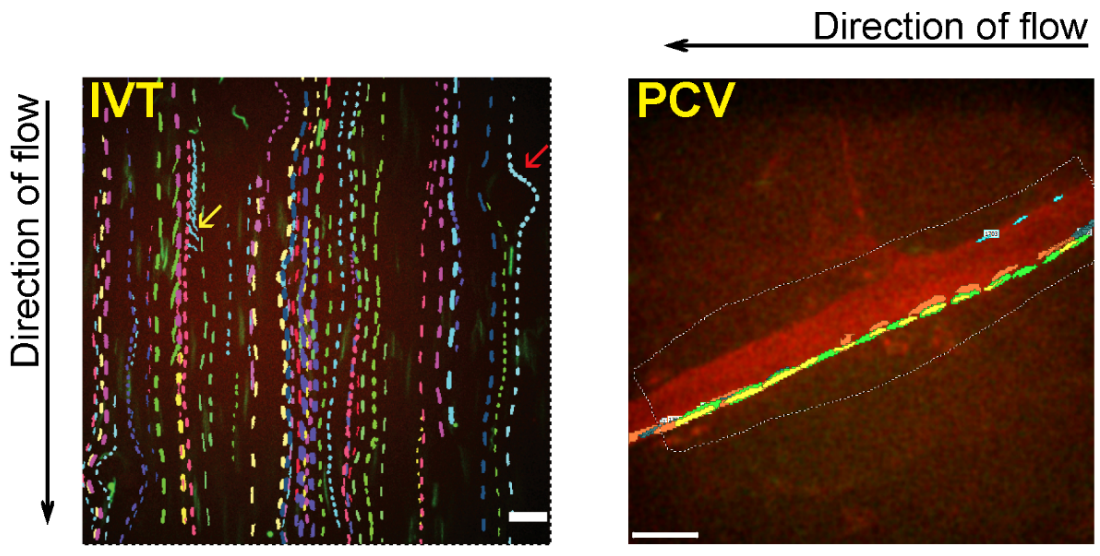

Figure 17. Trajectories of individual $B$. burgdorferi (different colours for each track) interacting with primary human endothelial cells in a flow chamber under postcapillary venule (PCV) shear stress conditions (IVT), and with a dermal PCV in a live mouse (PCV: IVM). Scale bars: IVT, $13 \mu \mathrm{m} ; \mathrm{PCV}, 32 \mu \mathrm{m}$. Endothelial counterstain (red): plasma membrane dye (IVT), AlexaFluor 555-PECAM-1 antibody (PCV). Yellow and red arrows: $B$. burgdorferi interacting by one end only (yellow) and moving along edge of endothelial nucleus projecting above imaging plane (red). Image from (Ebady et al., 2016).

In vitro live cell imaging of $B$. burgdorferimammalian host interactions

IVM visualization of $B$. burgdorferi in murine skin and the dermal microvasculature in turn enabled the development and validation of in vitro fluorescent live cell imaging models of host-pathogen interactions in the skin dermis (fluorescent bacteria moving in gelatin matrices) (Figure 12) and dermal microvasculature (fluorescent bacteria interacting with fluorescent counterstained primary human endothelial cells in blood-vessel mimicking flow chambers) (Figure 17) (Harman et al., 2012; Ebady et al., 2016; Salo et al., 2016; Kao et al., 2017; Niddam et al., 2017). These methods typically rely on GFP-expressing B. burgdorferi, although bacteria labelled with a non-toxic live cell imaging membrane dye can also be used (Salo et al., 2016).

One of the biggest challenges for in vitro live imaging is keeping cells healthy and fully functional for the duration of experiments (Ettinger and Wittmann, 2014). Most live cell imaging experiments are performed in a medium that is hospitable to both bacteria and host cells (not always an easy task to find), and in a controlled physical environment where temperature, $\mathrm{pH}$ and sometimes humidity are stably maintained using specialized heaters, $\mathrm{CO}_{2}$ and humidity controllers. Flow chamber live cell imaging also requires syringe or peristaltic pumps capable of precise output at low flow rates. All equipment must be regularly calibrated since small linear changes in variables such as shear stress can have log-scale effects on host-pathogen interactions in settings such as flow chambers.

Real-time time lapse imaging can be performed using epifluorescence, spinning disk confocal or resonant scanner confocal microscopes equipped with electron multiplying charge-coupled device (EMCCD) cameras or high sensitivity photomultiplier tube (PMT) sensors. As for all live cell microscopy, including ex vivo imaging and IVM, phototoxicity and photobleaching can pose significant challenges for acquiring repeated high frame rate time courses at the same position, particularly for confocal laser light sources (Ettinger and Wittmann, 2014). Visualizing thin bacteria, such as $B$. burgdorferi, in fast-moving settings such as blood vessels and flow chambers requires prioritization of image acquisition speed over image brightness and "beauty", especially for confocal microscopy, which excludes out of focus light. Prioritization of speed over beauty and brightness is especially important for measuring properties such as length and diameter of moving cells, which can appear larger when captured using EMCCD cameras and longer exposure times that produce more visually appealing, bright images (Jonkman et al., 2020). For this reason, accurate quantitative imaging for automated particle tracking and large-scale data analysis performed for fast- 
moving bacteria such as $B$. burgdorferi depends on non-saturated imaging conditions. For quantitative imaging, universal image acquisition conditions must also be pre-established and uniformly applied for all experimental conditions, and recalibrated over time as lasers age and become less powerful, and each time a new batch of fluorescent counterstain reagents is prepared or purchased (Jost and Waters, 2019).

\section{Advantages \\ Ex vivo imaging in tick midguts and salivary glands after transmission to ticks or during transmission to mammals offers the only currently available method to directly observe $B$. burgdorferi-tick interactions at the single cell level. It has provided dynamic, localized insight into mechanisms of bacterial migration during tick transmission that would not be possible without direct observation at single cell resolution (Dunham-Ems et al., 2009; Dunham-Ems et al., 2012; Caimano et al., 2015).}

Host-mimicking live cell imaging-based model systems support detailed, single cell resolution kinetic, biophysical, biochemical and genetic analysis of host-pathogen interaction mechanisms that are faster, less costly and more easily manipulated than IVM in animals (Harman et al., 2012; Ebady et al., 2016; Salo et al., 2016; Kao et al., 2017; Niddam et al., 2017). They facilitate interpretation of hostpathogen interaction data because immune clearance, which confounds IVM studies in blood vessels (Moriarty et al., 2012; Kumar et al., 2015), is largely absent. In vitro live cell imaging systems also support investigation of $B$. burgdorferi interactions with human cells, and rapid capture of large datasets (>10,000 datapoints) that can be analyzed by powerful segmentation and bioinformatics approaches.

\section{Disadvantages}

Ex vivo imaging in tick midguts and salivary glands requires delicate dissection, particularly if nymphal ticks are used, and separates these organs from the rest of the tick body, possibly disrupting some aspects of their physiology and function. Capturing optical sections through the full thickness of some tissues (e.g. late stage post-blood meal midguts) depends on physical sectioning or long working range objectives. Time-lapse imaging of live $B$. burgdorferi in tick midguts and salivary glands is performed for a maximum of 1.5 hours after dissection and fluorescent labeling of tick cells, necessitating the use of different ticks for each time point during the $\sim 48-72$ hour feeding period (Dunham-Ems et al., 2009).

In vitro live cell imaging model systems must be validated using in vivo methods such as whole body and IVM imaging, and/or qPCR and cultivation-based measurement of bacterial burden. In vitro live cell imaging depends on a stable, well-controlled physical environment. Quantitative live cell imaging also requires carefully controlled and calibrated imaging conditions, and substantial training and quality control monitoring. Efficient extraction of maximal amounts of information from high frame rate live cell imaging experiments can necessitate development and validation of automated methods for particle tracking and analyzing large volumes of data, and relies heavily on coding and bioinformatics skills that can be challenging for more conventionally trained biologists and microbiologists to quickly master if they are not working in collaborative teams.

\section{Tick-B. burgdorferi interactions}

Live ex vivo imaging methods developed for dissected tick guts and salivary glands (DunhamEms et al., 2009) have provided crucial insight into the processes of initial $B$. burgdorferi colonization of the tick midgut during mammalian host to tick transmission, and subsequent dissemination from the tick midgut to salivary glands during tick to mammalian host transmission. Live ex vivo imaging shows that these processes are controlled by the RpoS regulon (Dunham-Ems et al., 2012; Caimano et al., 2015). In B. burgdorferi-colonized unfed ticks, a mesh of non-motile spirochetes covers the epithelial lining of the tick midgut (Dunham-Ems et al., 2009). As colonized ticks feed, this mesh advances basally as the epithelium proliferates and differentiates in response to the blood meal. Basally positioned spirochetes then become motile and enter the hemocoel to migrate to and enter the salivary glands, from which they are transmitted to mammalian hosts (Dunham-Ems et al., 2009) (Figure 16). Spirochetes deficient in the RpoS regulon transform into a reversible round body morphotype as the blood meal progresses, and cannot migrate to salivary glands (Dunham-Ems et al., 2012). After naïve ticks feed on infected mammalian hosts, gene regulation switches to an RpoS-off state that promotes formation of the non-motile mesh of bacteria on epithelial surfaces that is typical of unfed, midgut colonization (Caimano et al., 2015). 


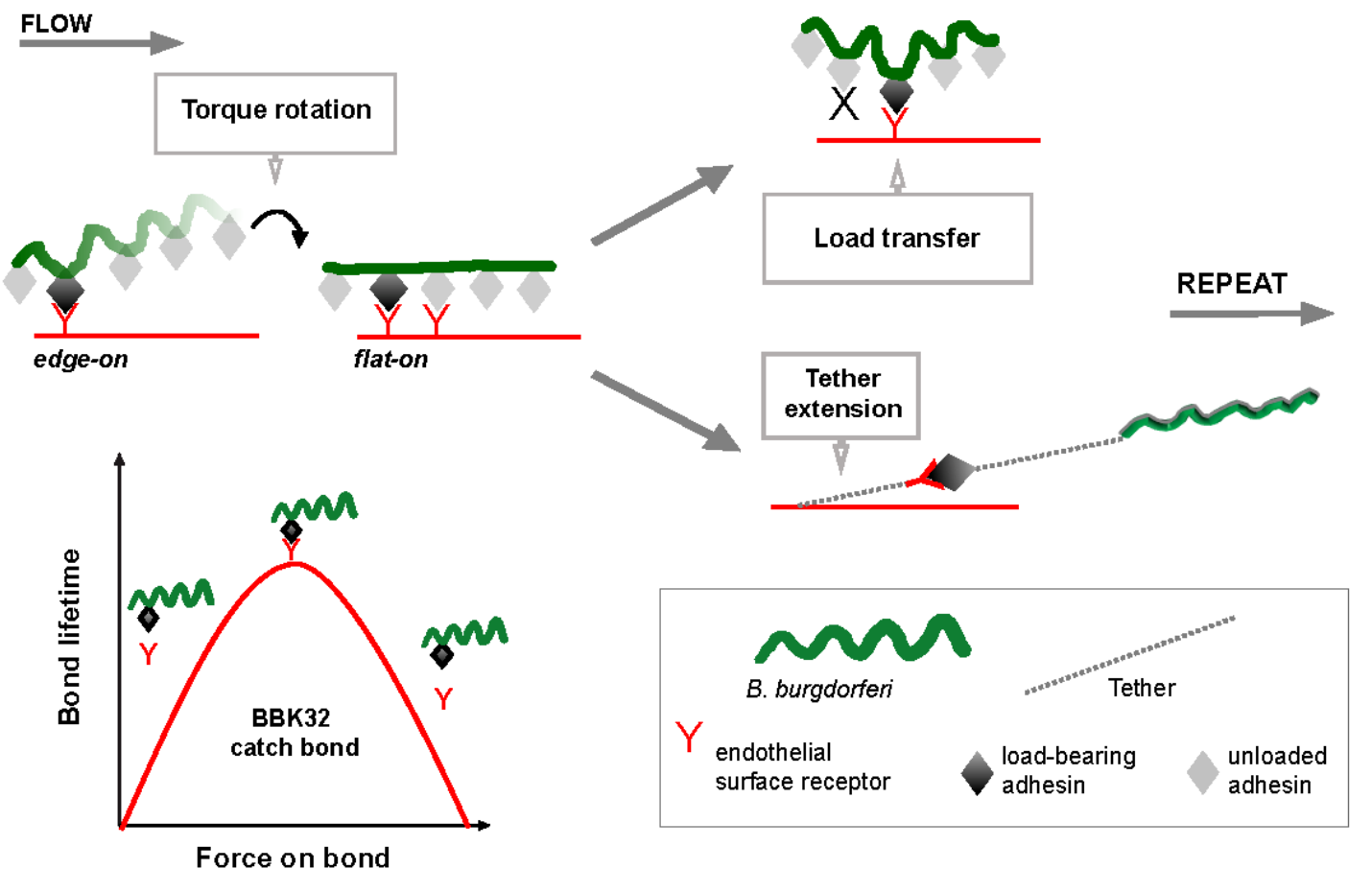

Figure 18. Under PCV shear stress conditions, slower moving "dragging" (untethered) B. burgdorferi is oriented edge-on to the endothelial surface and moves in a stepwise fashion, by transferring mechanical load from a single or coordinated, tightly clustered adhesion complex at the peak of the bacterial sine wave to another adhesion complex on the next sine wave peak. This process is facilitated by rotation of bacteria driven by flow-induced torque or bacterial motility. Faster-moving interactions are stabilized by tethers of unknown composition anchoring adhesion complexes and $B$. burgdorferi to the endothelial surface. Interactions are also stabilized by BBK32-dependent catch bonds, which are bonds that become longer lived as pulling force on the bond increases. Image from (Ebady et al., 2016).

\section{Shared human and mouse B. burgdorferi- endothelial interaction mechanisms}

Live cell imaging of $B$. burgdorferi interactions with primary human umbilical cord vein endothelial cells in flow chambers under postcapillary venule shear stress conditions reveals that basic interaction features and properties observed in dermal postcapillary venules (PCVs) of mice by IVM (Moriarty et al., 2008; Norman et al., 2008) are recapitulated with human endothelial cells in vitro (Ebady et al., 2016; Kao et al., 2017; Niddam et al., 2017). In both settings, B. burgdorferi exhibits tethering, dragging and stationary adhesion, tethering and dragging depend on $\mathrm{Fn}$, GAGs, the vascular adhesin BBK32 and at least one additional unidentified vascular adhesin, and bacterial strainspecific differences in interaction properties are conserved. Conversely, B. burgdorferi-endothelial interaction mechanisms first identified in flow chambers, such as transfer of mechanical load along a series of single adhesion complexes, were subsequently confirmed in vivo (Ebady et al., 2016). Identification of a $T$. pallidum adhesin supporting human endothelial interactions in flow chambers (pallilysin) led to confirmation of a role for this adhesin in dermal PCV interactions in mice (Kao et al., 2017). Thus, data derived from flow chamber model systems and human cells are useful for dissecting and predicting mouse vascular interaction mechanisms in vivo.

\section{Biomechanics of B. burgdorferi-endothelial interactions}

High speed particle tracking performed using large flow chamber and IVM datasets reveals that $B$. burgdorferi interactions with murine skin 


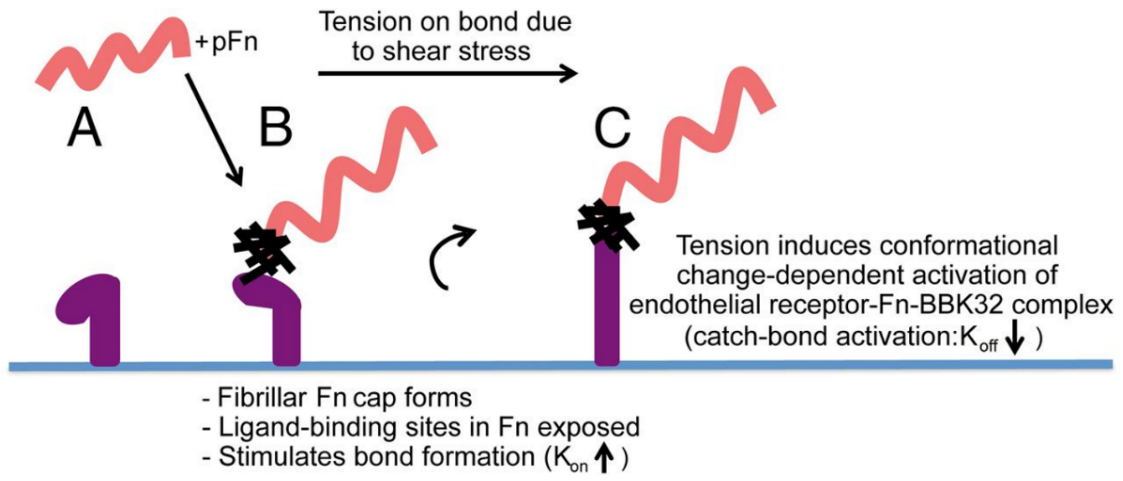

Figure 19. Proposed mechanism of plasma fibronectin ( $\mathrm{pFn}$ )-dependent $B$. burgdorferi-vascular interactions under physiological shear stress. Conformational changes and polymerization of $\mathrm{pFn}$ exposed to a BBK32-expressing bacterium $(A)$ exposes pFn binding sites for endothelial receptors and increases bond formation rate $\left(K_{\circ n}\right)(B)$. As bacteria are pushed/pulled by shear stress, stretching of the load-bearing adhesion complex induces additional force-induced conformational changes in adhesion complex components (BBK32, pFn, and endothelial receptors) to promote formation of longer-lived catch bonds and slower adhesion complex dissociation. Image from (Niddam et al., 2017).

microvessels and human endothelial cells in flow chambers occur by transfer of mechanical load along a series of single or tightly clustered adhesion complexes (Ebady et al., 2016), similar to the interaction mechanism used by leukocytes and platelets rolling along endothelial surfaces in PCVs (Begandt et al., 2017; Coenen et al., 2017) (Figure 17).

Leukocyte and platelet rolling interactions are stabilized by tethers, which are extendible membranous or filamentous structures that distribute (reduce) force imposed on load-bearing adhesion complexes (Begandt et al., 2017; Coenen et al., 2017). Particle tracking of $B$. burgdorferi-endothelial interactions in flow chambers and PCVs shows that these interactions are also stabilized by tethers, although the composition of these tethers remains unknown. These tethered interactions correspond to the faster-moving transient interactions referred to as "tethering" in prior observational IVM studies (Figure 18) (Ebady et al., 2016).

The slow interaction type identified as "dragging" in early IVM studies in fact corresponds to untethered bacteria that are oriented with their sine wave edgeon to endothelial surfaces, projecting up into flow (Figure 18) (Ebady et al., 2016). In untethered interactions, mechanical load is transferred from peak to peak of the sine wave, resulting in slowmoving step-wise incremental displacements equal to the distance between sine wave peaks $(\sim 2.83 \mu \mathrm{m})$.
Load-bearing bonds supporting untethered interactions are subjected to considerably more pulling force, due to the projection of bacteria up into flow. For human umbilical vein endothelial cells and murine dermal PCVs, the adhesin BBK32 stabilizes higher force interactions by a catch bond mechanism. Catch bonds are adhesive bonds that become longer-lived (more stable) under increasing pulling force, and are important for stabilization of other interaction types in the vasculature, including leukocyte and platelet rolling (Begandt et al., 2017; Coenen et al., 2017).

Flow chamber and particle-tracking approaches also show that the $B$. burgdorferi-endothelial catch bond interaction mechanism depends on soluble $\mathrm{Fn}$ recruited from blood plasma (plasma $\mathrm{Fn}, \mathrm{pFn}$ ), and not on insoluble $F n$ found on endothelial surfaces (Figure 19) (Niddam et al., 2017). BBK32-expressing bacteria induce $\mathrm{pFn}$ polymerization to form $\mathrm{pFn}$ caps positioned at the mechanical load-bearing ends of $B$. burgdorferi, stimulating formation of adhesion complexes with receptors on endothelial surfaces, and slowing dissociation of these complexes by a catch bond mechanism.

Interestingly, multiple flow chamber and IVM studies have found that adhesive bond forces supporting bacterial-endothelial interactions under PCV shear stress are sufficiently small that propulsive forces generated by $B$. burgdorferi motility should be large enough to modulate the forces imposed on these 
bonds, or even to break them (Ebady et al., 2016; Kao et al., 2017; Niddam et al., 2017). It is also possible that $B$. burgdorferi motility contributes to maintaining untethered interactions in an edge-on position relative to endothelial surfaces or bacterial rotation during interactions (Figure 18), or could contribute to the pulling forces driving $\mathrm{pFn}$ polymerization to the load-bearing ends of cells (Figure 19).

\section{Acknowledgements}

The authors would like to thank Alexia Belperron and Linda Bockenstedt for providing the image for Figure 10 and Björn Petri and $\mathrm{Xi}$ Tan for the image for Figure 15. Work in the Chaconas aboratory is supported by the Canadian Institutes of Health Research (PJT-153336) and the National Institute of Health (R01Al18799) and work in the Moriarty laboratory is supported by the Canadian Institutes of Health Research (PJT-159466) and the Natural Sciences and Engineering Research Council of Canada (RGPIN-401938-17). Research in the Skare laboratory is currently funded by NIAID Public Health Service grants Al131656 and Al146930 and research in the Hyde laboratory is currently funded by NIAID Public Health Service grants Al101740 and Al131656.

\section{References}

Adams, P.P., Flores Avile, C., and Jewett, M.W. (2017). A Dual Luciferase Reporter System for $B$. burgdorferi Measures Transcriptional Activity during Tick-Pathogen Interactions. Frontiers in cellular and infection microbiology 7, 225. https://doi.org/10.3389/ fcimb.2017.00225

Adams, P.P., Flores Avile, C., Popitsch, N., Bilusic, I., Schroeder, R., Lybecker, M., and Jewett, M.W. (2016). In vivo expression technology and $5^{\prime}$ end mapping of the Borrelia burgdorferi transcriptome identify novel RNAs expressed during mammalian infection. Nucleic Acids Res. https://doi.org/10.1093/nar/gkw1180

Andreu, N., Zelmer, A., and Wiles, S. (2011). Noninvasive biophotonic imaging for studies of infectious disease. FEMS Microbiol Rev 35, 360-394. https://doi.org/10.1111/j.1574-6976.2010.00252.x

Barthold, S.W., Sidman, C.L., and Smith, A.L. (1992). Lyme borreliosis in genetically resistant and susceptible mice with severe combined immunodeficiency. The American journal of tropical medicine and hygiene 47, 605-613. https://doi.org/https://doi.org/10.4269/ajtmh. 1992.47.605

Begandt, D., Thome, S., Sperandio, M., and Walzog, B. (2017). How neutrophils resist shear stress at blood vessel walls: molecular mechanisms, subcellular structures, and cell-cell interactions. J Leukoc Biol 102, 699-709. https://doi.org/10.1189/jlb. 3MR0117-026RR

Belperron, A.A., Mao, J., and Bockenstedt, L.K. (2018). Two Photon Intravital Microscopy of Lyme Borrelia in Mice. Methods Mol Biol 1690, 279-290. https://doi.org/10.1007/978-1-4939-7383-5_20

Blevins, J.S., Hagman, K.E., and Norgard, M.V. (2008). Assessment of decorin-binding protein A to the infectivity of Borrelia burgdorferi in the murine models of needle and tick infection. BMC microbiology 8,82 . https://doi.org/10.1186/1471-2180-8-82
Blevins, J.S., Revel, A.T., Smith, A.H., Bachlani, G.N., and Norgard, M.V. (2007). Adaptation of a luciferase gene reporter and lac expression system to Borrelia burgdorferi. Appl Environ Microbiol 73, 1501-1513. https://doi.org/https://doi.org/10.1128/AEM.02454-06

Bockenstedt, L.K., Gonzalez, D., Mao, J., Li, M., Belperron, A.A., and Haberman, A. (2014). What ticks do under your skin: two-photon intravital imaging of Ixodes scapularis feeding in the presence of the Lyme disease spirochete. Yale J Biol Med 87, 3-13.

Bockenstedt, L.K., Gonzalez, D.G., Haberman, A.M., and Belperron, A.A. (2012). Spirochete antigens persist near cartilage after murine Lyme borreliosis therapy. J Clin Invest 122, 2652-2660. https:// doi.org/10.1172/JCl58813

Bolz, D.D., Sundsbak, R.S., Ma, Y., Akira, S., Kirschning, C.J., Zachary, J.F., Weis, J.H., and Weis, J.J. (2004). MyD88 plays a unique role in host defense but not arthritis development in Lyme disease. J Immunol 173, 2003-2010. https://doi.org/https://doi.org/10.4049/ jimmunol.173.3.2003

Caimano, M.J., Dunham-Ems, S., Allard, A.M., Cassera, M.B., Kenedy, M., and Radolf, J.D. (2015). Cyclic di-GMP modulates gene expression in Lyme disease spirochetes at the tick-mammal interface to promote spirochete survival during the blood meal and tick-tomammal transmission. Infect Immun 83, 3043-3060. https://doi.org/ 10.1128/IAI.00315-15

Caimano, M.J., Eggers, C.H., Hazlett, K.R., and Radolf, J.D. (2004). RpoS is not central to the general stress response in Borrelia burgdorferi but does control expression of one or more essential virulence determinants. Infect Immun 72, 6433-6445. https://doi.org/ 10.1128/IAI.72.11.6433-6445.2004

Chan, K., Alter, L., Barthold, S.W., and Parveen, N. (2015). Disruption of bbe02 by Insertion of a Luciferase Gene Increases Transformation Efficiency of Borrelia burgdorferi and Allows Live Imaging in Lyme Disease Susceptible C3H Mice. PLoS One 10, e0129532. https:// doi.org/10.1371/journal.pone.0129532

Coenen, D.M., Mastenbroek, T.G., and Cosemans, J. (2017). Platelet interaction with activated endothelium: mechanistic insights from microfluidics. Blood 130, 2819-2828. https://doi.org/10.1182/ blood-2017-04-780825

Comstock, L.E., and Thomas, D.D. (1989). Penetration of endothelial cell monolayers by Borrelia burgdorferi. Infect Immun 57, 1626-1628. https://doi.org/https://doi.org/10.1128//AI.57.5.1626-1628.1989

Comstock, L.E., and Thomas, D.D. (1991). Characterization of Borrelia burgdorferi invasion of cultured endothelial cells. Microb Pathog 10 137-148. https://doi.org/https://doi.org/10.1016/0882-4010(91)90074k

Contag, C.H., Contag, P.R., Mullins, J.I., Spilman, S.D., Stevenson, D.K., and Benaron, D.A. (1995). Photonic detection of bacterial pathogens in living hosts. Mol Microbiol 18, 593-603. https://doi.org/ 10.1111/j.1365-2958.1995.mmi_18040593.x

Dunham-Ems, S.M., Caimano, M.J., Eggers, C.H., and Radolf, J.D. (2012). Borrelia burgdorferi requires the alternative sigma factor RpoS for dissemination within the vector during tick-to-mammal transmission. PLoS Pathog 8, e1002532. https://doi.org/https:// doi.org/10.1371/journal.ppat.1002532

Dunham-Ems, S.M., Caimano, M.J., Pal, U., Wolgemuth, C.W., Eggers, C.H., Balic, A., and Radolf, J.D. (2009). Live imaging reveals a biphasic mode of dissemination of Borrelia burgdorferi within ticks. Clin Invest 119, 3652-3665. https://doi.org/https://doi.org/10.1172/ JCl39401

Ebady, R., Niddam, A.F., Boczula, A.E., Kim, Y.R., Gupta, N., Tang, T.T., Odisho, T., Zhi, H., Simmons, C.A., Skare, J.T., et al. (2016). Biomechanics of Borrelia burgdorferi Vascular Interactions. Cell Rep. https://doi.org/10.1016/j.celrep.2016.08.013

Ellis, T.C., Jain, S., Linowski, A.K., Rike, K., Bestor, A., Rosa, P.A. Halpern, M., Kurhanewicz, S., and Jewett, M.W. (2013). In vivo expression technology identifies a novel virulence factor critical for 
Borrelia burgdorferi persistence in mice. PLoS Pathog 9, e1003567. https://doi.org/https://doi.org/10.1371/journal.ppat.1003567

Filippi, M.D. (2019). Neutrophil transendothelial migration: updates and new perspectives. Blood. https://doi.org/10.1182/ blood-2018-12-844605

Grabowski, J.M., Offerdahl, D.K., and Bloom, M.E. (2018). The Use of Ex Vivo Organ Cultures in Tick-Borne Virus Research. ACS Infect Dis 4, 247-256. https://doi.org/10.1021/acsinfecdis.7b00274

Grimm, D., Tilly, K., Byram, R., Stewart, P.E., Krum, J.G., Bueschel, D.M., Schwan, T.G., Policastro, P.F., Elias, A.F., and Rosa, P.A. (2004). Outer-surface protein $C$ of the Lyme disease spirochete: a protein induced in ticks for infection of mammals. Proc Natl Acad Sci U S A 101, 3142-3147. https://doi.org/https://doi.org/10.1073/pnas. 0306845101

Hammer, B., Moter, A., Kahl, O., Alberti, G., and Gobel, U.B. (2001). Visualization of Borrelia burgdorferi sensu lato by fluorescence in situ hybridization (FISH) on whole-body sections of Ixodes ricinus ticks and gerbil skin biopsies. Microbiology 147, 1425-1436. https://doi.org/ 10.1099/00221287-147-6-1425

Harman, M.W., Dunham-Ems, S.M., Caimano, M.J., Belperron, A.A., Bockenstedt, L.K., Fu, H.C., Radolf, J.D., and Wolgemuth, C.W. (2012). The heterogeneous motility of the Lyme disease spirochete in gelatin mimics dissemination through tissue. Proc Natl Acad Sci U S A 109, 3059-3064. https://doi.org/https://doi.org10.1073/pnas. 1114362109

Hubner, A., Yang, X., Nolen, D.M., Popova, T.G., Cabello, F.C., and Norgard, M.V. (2001). Expression of Borrelia burgdorferi OspC and DbpA is controlled by a RpoN-RpoS regulatory pathway. Proc Natl Acad Sci U S A 98, 12724-12729. https://doi.org/https:// doi.org10.1073/pnas.231442498

Hutchens, M., and Luker, G.D. (2007). Applications of bioluminescence imaging to the study of infectious diseases. Cell Microbiol 9, 2315-2322. https://doi.org/10.1111/j.1462-5822.2007.00995.x

Hyde, J.A., and Skare, J.T. (2018). Detection of Bioluminescent Borrelia burgdorferi from In Vitro Cultivation and During Murine Infection. Methods Mol Biol 1690, 241-257. https://doi.org/ 10.1007/978-1-4939-7383-5_18

Hyde, J.A., Weening, E.H., Chang, M., Trzeciakowski, J.P., Hook, M., Cirillo, J.D., and Skare, J.T. (2011). Bioluminescent imaging of Borrelia burgdorferi in vivo demonstrates that the fibronectin-binding protein BBK32 is required for optimal infectivity. Mol Microbiol 82, 99-113. https://doi.org/https://doi.org/10.1111/j.1365-2958.2011. 07801.x

Jenkins, D.E., Oei, Y., Hornig, Y.S., Yu, S.F., Dusich, J., Purchio, T., and Contag, P.R. (2003). Bioluminescent imaging (BLI) to improve and refine traditional murine models of tumor growth and metastasis. Clin Exp Metastasis 20, 733-744. https://doi.org/10.1023/b:clin. 0000006815.49932 .98

Jost, A.P., and Waters, J.C. (2019). Designing a rigorous microscopy experiment: Validating methods and avoiding bias. J Cell Biol 218, 1452-1466. https://doi.org/10.1083/jcb.201812109

Kao, W.A., Petrosova, H., Ebady, R., Lithgow, K.V., Rojas, P., Zhang, Y., Kim, Y.E., Kim, Y.R., Odisho, T., Gupta, N., et al. (2017). Identification of Tp0751 (Pallilysin) as a Treponema pallidum Vascular Adhesin by Heterologous Expression in the Lyme disease Spirochete. Sci Rep 7, 1538. https://doi.org/10.1038/s41598-017-01589-4

Kumar, D., Ristow, L.C., Shi, M., Mukherjee, P., Caine, J.A., Lee, W.Y., Kubes, P., Coburn, J., and Chaconas, G. (2015). Intravital Imaging of Vascular Transmigration by the Lyme Spirochete: Requirement for the Integrin Binding Residues of the B. burgdorferi P66 Protein. PLoS Pathog 11, e1005333. https://doi.org/10.1371/journal.ppat.1005333

Larson, A.M. (2011). Multiphoton microscopy. Nature Photonics 5, 1. https://doi.org/https://doi.org/10.1038/nphoton.an.2010.2

Laschke, M.W., Kerdudou, S., Herrmann, M., and Menger, M. (2005). Intravital fluorescence microscopy: a novel tool for the study of the interaction of Staphylococcus aureus with the microvascular endothelium in vivo. J Infect Dis 191, 435-443. https://doi.org/https:// doi.org10.1086/427193

Lee, W.Y., Moriarty, T.J., Wong, C.H., Zhou, H., Strieter, R.M., van Rooijen, N., Chaconas, G., and Kubes, P. (2010). An intravascular immune response to Borrelia burgdorferi involves Kupffer cells and iNKT cells. Nat Immunol 11, 295-302. https://doi.org/https://doi.org/ 10.1038/ni.1855

Lee, W.Y., Sanz, M.J., Wong, C.H., Hardy, P.O., Salman-Dilgimen, A., Moriarty, T.J., Chaconas, G., Marques, A., Krawetz, R., Mody, C.H., et al. (2014). Invariant natural killer $T$ cells act as an extravascular cytotoxic barrier for joint-invading Lyme Borrelia. Proc Natl Acad Sci U S A 111, 13936-13941. https://doi.org/https://doi.org/10.1073/pnas. 1404769111

Li, X., Liu, X., Beck, D.S., Kantor, F.S., and Fikrig, E. (2006). Borrelia burgdorferi lacking BBK32, a fibronectin-binding protein, retains full pathogenicity. Infect Immun 74, 3305-3313. https://doi.org/https:// doi.org/10.1128/IAl.02035-05

Lienenklaus, S., Cornitescu, M., Zietara, N., Lyszkiewicz, M., Gekara, N., Jablonska, J., Edenhofer, F., Rajewsky, K., Bruder, D., Hafner, M., et al. (2009). Novel reporter mouse reveals constitutive and inflammatory expression of IFN-beta in vivo. J Immunol 183, 3229-3236. https://doi.org/10.4049/immunol.0804277

Lin, Y.P., Tan, X., Caine, J.A., Castellanos, M., Chaconas, G., Coburn, J., and Leong, J.M. (2020). Strain-specific joint invasion and colonization by Lyme disease spirochetes is promoted by outer surface protein C. PLoS Pathog 16, e1008516. https://doi.org/ 10.1371/journal.ppat.1008516

Liu, N., Montgomery, R.R., Barthold, S.W., and Bockenstedt, L.K. (2004). Myeloid differentiation antigen 88 deficiency impairs pathogen clearance but does not alter inflammation in Borrelia burgdorferiinfected mice. Infect Immun 72, 3195-3203. https://doi.org/10.1128/ IAl.72.6.3195-3203.2004

Masedunskas, A., Milberg, O., Porat-Shliom, N., Sramkova, M., Wigand, T., Amornphimoltham, P., and Weigert, R. (2012). Intravital microscopy: a practical guide on imaging intracellular structures in live animals. Bioarchitecture 2, 143-157. https://doi.org/10.4161/bioa. 21758

Medina-Perez, D.N., Wager, B., Troy, E., Gao, L., Norris, S.J., Lin, T., Hu, L., Hyde, J.A., Lybecker, M., and Skare, J.T. (2020). The intergenic small non-coding RNA ittA is required for optimal infectivity and tissue tropism in Borrelia burgdorferi. PLoS Pathog 16, e1008423. https://doi.org/10.1371/journal.ppat.1008423

Moriarty, T.J., Norman, M.U., Colarusso, P., Bankhead, T., Kubes, P., and Chaconas, G. (2008). Real-time high resolution 3D imaging of the lyme disease spirochete adhering to and escaping from the vasculature of a living host. PLoS Pathog 4, e1000090. https:// doi.org/https://doi.org/10.1371/journal.ppat.1000090

Moriarty, T.J., Shi, M., Lin, Y.P., Ebady, R., Zhou, H., Odisho, T., Hardy, P.O., Salman-Dilgimen, A., Wu, J., Weening, E.H., et al. (2012). Vascular binding of a pathogen under shear force through mechanistically distinct sequential interactions with host macromolecules. Mol Microbiol 86, 1116-1131. https://doi.org/https:// doi.org/10.1111/mmi.12045

Motaleb, M.A., Liu, J., and Wooten, R.M. (2015). Spirochetal motility and chemotaxis in the natural enzootic cycle and development of Lyme disease. Current Opinion in Microbiology 28, 106-113. https://doi.org/ http://dx.doi.org/10.1016/j.mib.2015.09.006

Niddam, A.F., Ebady, R., Bansal, A., Koehler, A., Hinz, B., and Moriarty, T.J. (2017). Plasma fibronectin stabilizes Borrelia burgdorferiendothelial interactions under vascular shear stress by a catch-bond mechanism. Proc Natl Acad Sci U S A 114, E3490-E3498. https:// doi.org/10.1073/pnas.1615007114

Norman, M.U., Moriarty, T.J., Dresser, A.R., Millen, B., Kubes, P., and Chaconas, G. (2008). Molecular mechanisms involved in vascular 
interactions of the Lyme disease pathogen in a living host. PLoS Pathog 4, e1000169. https://doi.org/https://doi.org/10.1371/ journal.ppat.1000169

Novak, E.A., Sekar, P., Xu, H., Moon, K.H., Manne, A., Wooten, R.M. and Motaleb, M.A. (2016). The Borrelia burgdorferi CheY3 Response Regulator is Essential for Chemotaxis and Completion of its Natural Infection Cycle. Cell Microbiol. https://doi.org/10.1111/cmi.12617

Oreopoulos, J., Berman, R., and Browne, M. (2014). Spinning-disk confocal microscopy: present technology and future trends. Methods in Cell Biology 123, 153-175. https://doi.org/10.1016/ B978-0-12-420138-5.00009-4.

Ouyang, Z., Narasimhan, S., Neelakanta, G., Kumar, M., Pal, U., Fikrig, E., and Norgard, M.V. (2012). Activation of the RpoN-RpoS regulatory pathway during the enzootic life cycle of Borrelia burgdorferi. BMC microbiology 12, 44. https://doi.org/https://doi.org/ 10.1186/1471-2180-12-44

Radolf J.D. and Samuels, D.S. (2021). Lyme Disease and Relapsing Fever Spirochetes: Genomics, Molecular Biology, Host Interactions and Disease Pathogenesis (Norfolk, UK: Caister Academic Press). https://doi.org/10.21775/9781913652616

Rehemtulla, A., Stegman, L.D., Cardozo, S.J., Gupta, S., Hall, D.E., Contag, C.H., and Ross, B.D. (2000). Rapid and quantitative assessment of cancer treatment response using in vivo bioluminescence imaging. Neoplasia 2, 491-495. https://doi.org/ 10.1038/sj.neo.7900121

Sadikot, R.T., Wudel, L.J., Jansen, D.E., Debelak, J.P., Yull, F.E., Christman, J.W., Blackwell, T.S., and Chapman, W.C. (2002). Hepatic cryoablation-induced multisystem injury: bioluminescent detection of NF-kappaB activation in a transgenic mouse model. J Gastrointest Surg 6, 264-270. https://doi.org/10.1016/s1091-255x(01)00064-6

Salo, J., Pietikainen, A., Soderstrom, M., Auvinen, K., Salmi, M., Ebady, R., Moriarty, T.J., Viljanen, M.K., and Hytonen, J. (2016). FlowTolerant Adhesion of a Bacterial Pathogen to Human Endothelial Cells Through Interaction With Biglycan. J Infect Dis 213, 1623-1631. https://doi.org/10.1093/infdis/jiw003

Saputra, E.P., Trzeciakowski, J.P., and Hyde, J.A. (2020). Borrelia burgdorferi spatiotemporal regulation of transcriptional regulator bosR and decorin binding protein during murine infection. Sci Rep 10, 12534. https://doi.org/10.1038/s41598-020-69212-7

Secklehner, J., Lo Celso, C., and Carlin, L.M. (2017). Intravital microscopy in historic and contemporary immunology. Immunol Cell Biol 95, 506-513. https://doi.org/10.1038/icb.2017.25

Seshu, J., Esteve-Gassent, M.D., Labandeira-Rey, M., Kim, J.H., Trzeciakowski, J.P., Hook, M., and Skare, J.T. (2006). Inactivation of the fibronectin-binding adhesin gene bbk32 significantly attenuates the infectivity potential of Borrelia burgdorferi. Mol Microbiol 59, 1591-1601. https://doi.org/https://doi.org/10.1111/j.1365-2958.2005.05042.x

Shi, Y., Xu, Q., McShan, K., and Liang, F.T. (2008). Both decorin-binding proteins $A$ and $B$ are critical for the overall virulence of Borrelia burgdorferi. Infect Immun 76, 1239-1246. https://doi.org/10.1128/IAI. 00897-07

Skare, J.T., Shaw, D.K., Trzeciakowski, J.P., and Hyde, J.A. (2016). In Vivo Imaging Demonstrates That Borrelia burgdorferi ospC Is Uniquely Expressed Temporally and Spatially throughout Experimental Infection. PLoS One 11, e0162501. https://doi.org/ 10.1371/journal.pone.0162501

Sultan, S.Z., Manne, A., Stewart, P.E., Bestor, A., Rosa, P.A., Charon, N.W., and Motaleb, M.A. (2013). Motility is crucial for the infectious life cycle of Borrelia burgdorferi. Infect Immun 81, 2012-2021. https:// doi.org/10.1128/IAI.01228-12
Sultan, S.Z., Sekar, P., Zhao, X., Manne, A., Liu, J., Wooten, R.M., and Motaleb, M.A. (2015). Motor rotation is essential for the formation of the periplasmic flagellar ribbon, cellular morphology, and Borrelia burgdorferi persistence within Ixodes scapularis tick and murine hosts. Infect Immun 83, 1765-1777. https://doi.org/10.1128/iai. 03097-14

Szczepanski, A., Furie, M., Benach, J., Lane, B., and Fleit, H. (1990). Interaction between Borrelia burgdorferi and endothelium in vitro. $J$ Clin Invest 85, 1637-1647. https://doi.org/https://doi.org/10.1172/ JCl114615

Takacs, C.N., Kloos, Z.A., Scott, M., Rosa, P.A., and Jacobs-Wagner, C. (2018). Fluorescent Proteins, Promoters, and Selectable Markers for Applications in the Lyme Disease Spirochete Borrelia burgdorferi. Appl Environ Microbiol 84. https://doi.org/10.1128/AEM.01824-18

Tan, X., Petri, B., and Chaconas, G. (2020). The Lyme disease spirochete hijacks the host immune system to extravasate through the microvasculature. Manuscript submitted.

Tilly, K., Bestor, A., Dulebohn, D.P., and Rosa, P.A. (2009). OspCindependent infection and dissemination by host-adapted Borrelia burgdorferi. Infect Immun 77, 2672-2682. https://doi.org/10.1128/IAI 01193-08

Tilly, K., Krum, J.G., Bestor, A., Jewett, M.W., Grimm, D., Bueschel, D., Byram, R., Dorward, D., Vanraden, M.J., Stewart, P., et al. (2006) Borrelia burgdorferi OspC protein required exclusively in a crucial early stage of mammalian Infection. Infect Immun 74, 3554-3564 https://doi.org/https://doi.org/10.1128//AI.01950-05

Wager, B., Shaw, D.K., Groshong, A.M., Blevins, J.S., and Skare, J.T. (2015). BB0744 Affects Tissue Tropism and Spatial Distribution of Borrelia burgdorferi. Infect Immun 83, 3693-3703. https://doi.org/ 10.1128/IAI.00828-15

Waidmann, M.S., Bleichrodt, F.S., Laslo, T., and Riedel, C.U. (2011). Bacterial luciferase reporters: the Swiss army knife of molecular biology. Bioeng Bugs 2, 8-16. https://doi.org/10.4161/bbug.2.1.13566

Wang, G., Ma, Y., Buyuk, A., McClain, S., Weis, J.J., and Schwartz, I. (2004). Impaired host defense to infection and Toll-like receptor 2independent killing of Borrelia burgdorferi clinical isolates in TLR2 deficient $\mathrm{C} 3 \mathrm{H} / \mathrm{HeJ}$ mice. FEMS Microbiol Lett 231, 219-225. https:/ doi.org/10.1016/S0378-1097(03)00960-1

Weening, E.H., Parveen, N., Trzeciakowski, J.P., Leong, J.M., Hook, M., and Skare, J.T. (2008). Borrelia burgdorferi lacking DbpBA exhibits an early survival defect during experimental infection. Infect Immun 76 5694-5705. https://doi.org/10.1128/IAI.00690-08

Wormser, G.P. (2006). Hematogenous dissemination in early Lyme disease. Wien Klin Wochenschr 118, 634-637. https://doi.org/https:// doi.org/10.1007/s00508-006-0688-9

Xu, T., Close, D., Handagama, W., Marr, E., Sayler, G., and Ripp, S. (2016). The Expanding Toolbox of In Vivo Bioluminescent Imaging. Front Oncol 6, 150. https://doi.org/10.3389/fonc.2016.00150

Yang, X.F., Lybecker, M.C., Pal, U., Alani, S.M., Blevins, J., Revel, A.T., Samuels, D.S., and Norgard, M.V. (2005). Analysis of the ospC regulatory element controlled by the RpoN-RpoS regulatory pathway in Borrelia burgdorferi. J Bacteriol 187, 4822-4829. https://doi.org/ https://doi.org/10.1128/JB.187.14.4822-4829.2005

Zhang, W., Contag, P.R., Madan, A., Stevenson, D.K., and Contag, C.H. (1999). Bioluminescence for biological sensing in living mammals Advances in experimental medicine and biology 471, 775-784. https:// doi.org/10.1007/978-1-4615-4717-4_89

Zhi, H., Weening, E.H., Barbu, E.M., Hyde, J.A., Hook, M., and Skare, J.T. (2015). The BBA33 lipoprotein binds collagen and impacts Borrelia burgdorferi pathogenesis. Mol Microbiol 96, 68-83. https:// doi.org/10.1111/mmi.12921 
